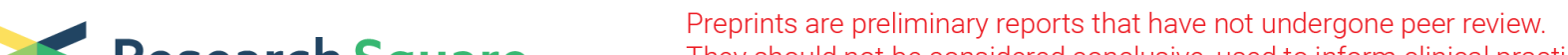 $\begin{array}{ll}\text { Research Square } & \begin{array}{l}\text { They should not be considered conclusive, used to inform clinical practice, } \\ \text { or referenced by the media as validated information. }\end{array}\end{array}$
}

\section{The long non-coding RNA 'TRASH' is essential for cell survival in MAPK-driven melanoma}

\author{
Valentin Feichtenschlager \\ University of California San Francisco

\section{Linan Chen} \\ University of California San Francisco \\ Yixuan Zheng \\ University of California San Francisco

\section{Wilson Ho} \\ University of California San Francisco

\section{Martina Sanlorenzo} \\ University of California San Francisco \\ Igor Vujic
}

Department of Dermatology and Venerology, Medical Institution Rudolfstiftung, Vienna, Austria

\section{Eleanor Fewings}

University of California San Francisco

\section{Albert Lee}

University of California San Francisco

\section{Christopher Chen}

University of California San Francisco

\section{Kevin Lin}

University of California San Francisco

\section{Ciara Callanan}

University of California San Francisco

\section{Marin Vujic}

University of California San Francisco

\section{Yeonjoo Hwang}

University of California, San Francisco

\section{Kevin Lai}

University of California San Francisco

\section{Stephanie Chen}

University of California San Francisco

\section{Thuan Nguyen}

University of California San Francisco

\section{Denise Muñoz}


University of California at San Francisco

\section{Yoshinori Kohwi}

Christian Posch

Technical University of Munich

\section{Adil Daud}

UCSF https://orcid.org/0000-0002-6617-8421

\section{Klemens Rappersberger}

Department of Dermatology and Venerology, Medical Institution Rudolfstiftung, Vienna, Austria

\section{Terumi Kohwi-Shigematsu}

Lawrence Berkeley National Laboratory

Jean-Philippe Coppé

University of California at San Francisco

Susana Ortiz-Urda ( $\nabla$ Susana.Ortiz@ucsf.edu)

University of California San Francisco

\section{Article}

Keywords:

Posted Date: February 11th, 2022

DOI: https://doi.org/10.21203/rs.3.rs-1297358/v1

License: (c) (i) This work is licensed under a Creative Commons Attribution 4.0 International License.

Read Full License 
Title Page.

Authors:

Valentin Feichtenschlager ${ }^{1,5}$, Linan Chen ${ }^{1}$, Yixuan James Zheng ${ }^{1,8}$, Wilson $\mathrm{Ho}^{1}$, Martina Sanlorenzo ${ }^{1}$, Igor Vujic $^{1,5,7}$, Eleanor Fewings ${ }^{1}$, Albert Lee ${ }^{1}$, Christopher Chen ${ }^{1}$, Kevin Lin $^{1}$, Ciara Callanan ${ }^{1}$, Marin Vujic ${ }^{1,5}$, Yeonjoo Hwang ${ }^{4}$, Kevin Lai ${ }^{1}$, Stephanie Chen ${ }^{1}$, Thuan Nguyen ${ }^{1}$, Denise P Muñoz ${ }^{4}$, Yoshinori Kohwi ${ }^{2}$, Christian Posch ${ }^{1,5,6,7}$, Adil Daud ${ }^{1}$, Klemens Rappersberger ${ }^{5}$, Terumi Kohwi-Shigematsu ${ }^{2}$, Jean-Philippe Coppé ${ }^{3}$, Susana Ortiz-Urda ${ }^{1 *}$

\section{Affiliations:}

${ }^{1}$ Department of Dermatology, Mt Zion Cancer Research Center, University of California San Francisco, San Francisco, CA, USA

${ }^{2}$ Department of Orofacial Science, Health Science West, University of California San Francisco School of Dentistry, San Francisco, CA, USA

${ }^{3}$ Department of Laboratory Medicine, Helen Diller Family Comprehensive Cancer Center, University of California San Francisco, San Francisco, CA, USA

${ }^{4}$ Department of Hematology-Oncology, Helen Diller Family Comprehensive Cancer Center, University of California San Francisco, San Francisco, CA, USA

${ }^{5}$ Department of Dermatology, Clinic Landstrasse Vienna, Academic Teaching Hospital, Medical University Vienna, Vienna, Austria

${ }^{6}$ Department of Dermatology and Allergy, School of Medicine, German Cancer Consortium (DKTK), Technical University of Munich, Munich, Germany.

${ }^{7}$ Sigmund Freud Private University, Faculty of Medicine, Vienna, Austria 
${ }^{8}$ School of Medicine, University of California San Francisco, San Francisco, CA, USA

*Corresponding Author.

Contact Information:

Susana Ortiz-Urda,

Department of Dermatology,

Mt Zion Cancer Research Center,

2340 Sutter Street, Room N419,

University of California San Francisco,

San Francisco, CA, USA

Tel.: 1-650-224-8265

Email: Susana.Ortiz@ucsf.edu 


\section{The long non-coding RNA 'TRASH' is essential for cell survival in MAPK-driven melanoma}

\section{Main Text:}

\section{Abstract:}

MAPK-pathway up-regulation is responsible for over $40 \%$ of human cancer cases. Finding effective therapeutic targets for melanoma therapy continues to be a challenge due to drug resistance. Using a computational and experimental pipeline, we discovered the nuclear enriched long non-coding RNA (IncRNA) TRASH, which is induced upon MAPK-pathway-activation in melanoma. LncRNA hold essential regulatory functions in many cancer types. TRASH-targeting Antisense Oligonucleotides (TRASH-ASOs) greatly reduced cell-growth of melanoma cells in culture and systemic TRASH-ASO treatment significantly inhibited tumor growth of melanoma cell-line and patient-derived tumor xenografts without apparent side effects. We found that TRASH is essential for protein stability of the MAPKpathway-regulating and apoptosis-inhibiting oncogene hnRNPA2/B1. TRASH knockdown induced apoptosis by down-regulating anti-apoptotic kinase activity and pro-survival signaling pathways. Compared to the well-studied oncogenic IncRNA MALAT1, the unique feature of TRASH is that it governs tumor cell-survival through maintaining activity of anti-apoptotic kinases and pro-survival signaling pathways in melanoma cells. TRASH-ASO treatment can bypass MEK-inhibitor (MEKi) resistance, and unlike MEKi, it does not induce early-onset treatment resistance. Furthermore, TRASH-ASO and MEKi combinations confer synergistic effects in melanoma treatment. Our findings show that TRASH-ASO treatment presents promising features for long lasting RNA-targeting melanoma therapy. 


\section{Introduction:}

Melanoma is the deadliest form of skin cancer and its incidence is rising. ${ }^{1}$ Most solid tumors, including melanoma, harbor MAPK-pathway activating mutations. These signaling cascades ultimately regulate cell proliferation, survival, and apoptosis. Targeting essential components of the MAPK-pathway such as BRAF and MEK kinases has yielded effective melanoma therapy during the last two decades. ${ }^{2,3}$ The antitumor effect of agents like BRAF and MEK-inhibitors relies on the stimulation of apoptosis activating pathways. ${ }^{4}$ Apoptosis is a highly regulated mechanism of programmed cell death characterized by caspase-dependent dissolution of intracellular components. It can be induced through extrinsic or intrinsic pathways, leading to activation of effector caspases, like caspase-3 \& -7 , which function as executioner proteases that lead to proteolysis and cell death. The mechanisms of apoptosis involve a complex machinery of interlocking processes that can be dysregulated in cancer. ${ }^{5}$ An example for an apoptosis-inhibiting oncogene is hnRNPA2/B1, which is over-expressed in many cancers. Its mechanisms involve interactions with oncogenic IncRNAs, modulation of the MAPK-, and PI3K/AKT-pathways, and regulation of alternative splicing. ${ }^{6-10}$

An ongoing focus in clinical oncology is the development of therapeutic agents that promote apoptosis to eradicate cancer cells. ${ }^{5}$ However, patients with initial or acquired treatment resistance point toward the limitations of existing cancer therapy. Therefore, an increased armament of anti-cancer therapeutics is urgently needed. ${ }^{11}$

A large fraction of the transcriptome remains untranslated and the majority of those transcripts are long non-coding RNAs (IncRNAs), defined as non-coding sequences longer than 200 nucleotides. ${ }^{12}$ LncRNAs are involved in oncogenesis through several mechanisms. They can regulate cancer-specific gene expression as splicing factors or through epigenetic histone modification. ${ }^{13}$ LncRNAs can also activate or stabilize proteins through binding to promote malignancy and affect drug resistance.${ }^{14-16}$ More and 
more IncRNA transcripts such as MALAT1, PANDAR or PCA3 are getting identified and characterized as important tumor suppressing or oncogenic regulators. ${ }^{17}$

In recent years, an increasing number of RNA-targeting Antisense Oligonucleotides (ASOs) have been brought to clinical trials and obtained FDA approval. ${ }^{18,19}$ In particular, emerging findings in IncRNAtargeted gene silencing show promising results. ${ }^{20}$

We explored a link of several IncRNAs to the MAPK-pathway and their relevance for melanoma cellsurvival and tumor progression. As a result, we identified the IncRNA TRASH (TRanscript $\underline{\text { ASsociated with }}$ HnRNPA2/B1) and its central role in melanoma biology. We found that TRASH is required for melanoma cell-survival and sustains melanoma cell-growth. TRASH knockdown mediated by Antisense Oligonucleotides (TRASH-ASOs) resulted in efficient suppression of anti-apoptotic kinase activity and promoted cell death in a broad panel of melanoma cell-lines, including melanoma resistant to MEKinhibition (MEKi). TRASH was found to bind to hnRNPA2/B1 and TRASH-ASO treatment led to concomitant down-regulation of hnRNPA2/B1 protein levels. In vitro evaluation of additional probable clinical features of TRASH-inhibition unveiled that TRASH-ASO treatment showed synergistic treatment effects in dual application with MEKi. In contrast to MEKi treatment, TRASH-ASO treatment did not lead to early-onset treatment resistance.

In mouse models of melanoma cell-line xenografts and patient-derived tumors, TRASH-ASO treatment induced apoptosis and strongly reduced tumor growth. In summary, these findings demonstrate the strong potential of TRASH-ASOs for the treatment of melanoma. 


\section{Results:}

Identification of MAPK-pathway-activation responsive IncRNAs in melanoma

The oncogene NRAS is an upstream regulator of the MAPK-pathway. ${ }^{21}$ NRAS-mutations are an early event in melanocytic tumorigenesis and NRAS-activation leads to activation of the downstream targets AKT and ERK. ${ }^{22-24}$ To identify IncRNA transcripts that respond to MAPK-pathway-activation, we introduced an NRAS ${ }^{\mathrm{Q} 61}$-mutant plasmid into primary human melanocytic cell-lines $\left(\mathrm{PHM}^{\mathrm{Q} 61}\right)$ via lentiviral transduction. Alongside this, an empty vector was transduced into a control group of primary human melanocytic cell-lines $\left(\mathrm{PHM}^{\mathrm{E}}\right)$. Successful transduction was confirmed through up-regulated levels of phosphorylated ERK and AKT (p-ERK and p-AKT) in PHM ${ }^{\mathrm{Q} 61}$ (Suppl. Fig. 1a). Activating NRAS-mutations like NRAS ${ }^{\mathrm{Q} 61}$ occur in benign nevi and additional transformations are needed to fully-unfold the malignant potential of melanocytes. ${ }^{25}$ No significant differences in cell proliferation could be measured comparing $\mathrm{PHM}^{\mathrm{Q} 61}$ and $\mathrm{PHM}^{\mathrm{e}}$, confirming that a sole $\mathrm{NRAS}^{\mathrm{Q61}}$-mutation is insufficient to equip melanocytic cell-lines with significant cell-growth characteristics (Suppl. Fig. 1a).

We present a schematic workflow overview of the combined in silico and in vitro processes to identify IncRNAs regulated in response to MAPK-pathway-activation which are essential for melanoma cellsurvival. (Fig. 1a) First, we compared paired-end non-poly A enriched 101-bp RNA-Seq data from PHM (no vector), $\mathrm{PHM}^{\mathrm{E}}, \mathrm{PHM}^{\mathrm{Q} 61}$, and two melanoma cell-lines (D04, MM415) harboring MAPK-pathway hyper-activating mutations. 197 genes were differently expressed (DE) and showed the same tendency in $\mathrm{PHM}^{\mathrm{Q} 61}$, D04, and MM415 when compared to standard melanocytes (PHM ${ }^{\mathrm{Q} 61} \Delta \mathrm{PHM}^{\mathrm{E}}$; D04 $\triangle \mathrm{PHM}$; M415 $\triangle$ PHM) (Fig. 1b-c, Suppl. Fig. 1b). 81 of the DE genes were up-regulated IncRNAs in all three comparisons. 24 of those IncRNAs were potentially clinically relevant as they were also expressed (FPKM-values $>0.2$ ) in $>90 \%$ of patient-derived NRAS mutant melanoma tumors from the Cancer Genome Atlas Skin Cutaneous Melanoma (TCGA-SKCM) dataset. (Fig. 1d, Suppl. Fig. 1c). We used small interfering RNA (siRNA) and esiRNA libraries (endoribonuclease prepared siRNA) to screen for IncRNAs 
essential for melanoma cell-survival. For 6 of the targets, RNAi led to significant cell-growth reduction in melanoma cell-lines, while no such impact could be observed in primary human melanocytic cell-lines.

Fig. 1e shows RNAi screening effects for silencing of the IncRNA AC004540.4, the transcript we identified as a top candidate for further investigations. These findings unveiled MAPK-activation responsive IncRNAs that are essential for melanoma cell-survival.

The IncRNA AC004540.4 (TRASH) is a nuclear regulator of hnRNPA2/B1 AC004540.4 is a IncRNA located on chromosome 7, expressed in two isoforms, and not conserved in other species (Suppl. Fig. 2a). Typically, the regulatory functions of IncRNAs are closely related to their subcellular localization. ${ }^{26}$ With IncRNAs primarily localized in the nucleus, the role of AC004540.4 in melanoma homeostasis was assessed by analysis of subcellular fractionations. We demonstrate that AC004540.4 is highly enriched in the nuclear compartment versus the cytoplasm in the melanoma cellline D04 (Fig. 2a). Nuclear enriched IncRNAs often exist in inefficiently spliced states. ${ }^{27}$ Using four different primer pairs for comparison of relative quantification of different regions of AC004540.4 through qRT-PCR revealed that exonic, intronic, and exon/intron transition regions of AC004540.4 were detectable in different quantities, indicating that AC004540.4 transcripts may exist to a certain extent in inefficient spliced states (Fig. 2b). Nuclear enriched IncRNA functions typically involve cis-regulation and co-expression of protein-coding genes that are located in close proximity. ${ }^{27}$ The closest coding gene to AC004540.4 on the same strand is the oncogene hnRNPA2/B1. HnRNPA2/B1 is part of the family of heterogeneous nuclear ribonucleoproteins (hnRNPs), a group of proteins that have at least one RNAbinding motif and regulate nucleic acid metabolism. ${ }^{28}$ HnRNPA2/B1 interacts with IncRNAs and exerts regulatory functions in MAPK-pathway signaling. ${ }^{6-10}$ Afterwards, we used the TCGA and The GenotypeTissue Expression (GTEx) project databases to study whether AC004540.4 and hnRNPA2/B1 have any functional relationship in patient derived melanoma and/or non-malignant skin biopsies. We explored 
the correlation of expression between both genes compared it to permutations of randomly chosen genes. Most notably, RNA-expression of AC004540.4 and hnRNPA2/B1 was significantly higher in melanoma (Fig 2c). The correlation of both genes was almost always significantly stronger in melanoma than the average correlation of each gene to 10 sets of random genes $(p<0.0510 / 10$ for AC004540.4 and 8/10 for hnRNPA2/B1). In contrast, in non-malignant skin samples, there were no significant differences in AC004540.4-, and hnRNPA2/B1-expression in any of the 20 comparisons (Suppl. Fig. 2b-e). Based on the gene expression correlation and the subsequently presented functional studies, we named

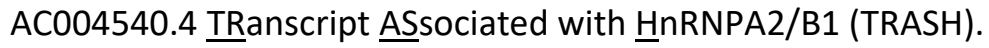

Synthetic nucleic acids such as siRNA and antisense oligonucleotides (ASOs) are frequently used laboratory methods for silencing IncRNA-expression. Several have proven to bear strong clinical value and received FDA and/or EMA approval for therapeutic approaches. ${ }^{20}$ Compared to siRNA, ASOmediated gene silencing is more effective for silencing nuclear localized IncRNAs and allows more chemical modification of synthetic nucleic acids to reduce undesirable side effects. ${ }^{29,30}$ ASO improvement with locked nucleic acids (LNAs) leads to more specificity and less toxicity. ${ }^{17}$ With the goal of clinical applicability in mind, we focused on LNA-improved ASOs for all further TRASH-knockdown experiments (TRASH-ASOs).

TRASH-ASO treatment did not significantly affect hnRNPA2/B1 transcript levels in D04 melanoma cells, indicating that hnRNPA2/B1 transcription does not depend on TRASH transcript levels (Fig. 2d). To investigate if TRASH expression regulates hnRNPA2/B1 protein expression, we examined protein levels of hnRNPA2/B1 1 and 2 days after TRASH-ASO treatment. By Immunoblot, hnRNPA2/B1 protein was found to be essentially undetected upon TRASH-inhibition (Fig. 2e). Although loss of hnRNPA2/B1 protein by TRASH-inhibition could be an indirect effect, our data suggests that TRASH either has an essential role for hnRNPA2/B1 protein translation or affects hnRNPA2/B1 protein stability by direct 
association in the nucleus. However, the former is unlikely as TRASH is highly enriched in the nucleus and hnRNPA2/B1 has a half-life of several days in primary human cells (Fig. 2a). ${ }^{31}$ To investigate if hnRNPA2/B1 protein associates with TRASH in the nucleus, we performed RIP-assay by pulling down hnRNPA2/B1 from melanoma cell lysate with the use of antibodies against hnRNPA2/B1 and examined RNA associated with the immunoprecipitated material. TRASH was successfully immunoprecipitated with hnRNPA2/B1 (shown by immunoblot, Fig. 2f) and it was enriched $>65$-fold compared to negative control pulldown with normal IgG. Therefore, TRASH and the protein hnRNPA2/B1 physically interact (Fig. 2f).

These findings indicate that up-regulation of TRASH and hnRNPA2/B1 transcription is a melanoma specific event and TRASH and hnRNPA2/B1 protein physically interact with each other. Considering prior studies demonstrating that IncRNAs can stabilize proteins and inhibit their degradation, TRASHexpression may be essential for maintaining stable hnRNPA2/B1 protein levels in melanoma. ${ }^{15}$

$\underline{\text { TRASH is an anti-apoptotic regulator in MAPK-driven melanoma. }}$

NRAS-, BRAF-, and c-KIT-mutations are MAPK-pathway activating mutations that frequently occur in melanoma patients. ${ }^{32-34}$ We investigated the effects of TRASH-inhibition on MAPK-driven melanoma by applying TRASH-ASO treatment to a repository of established and primary patient-derived melanoma cell-lines that harbor these mutations (see Methods). In comparison to non-targeting control ASO (Control-ASO) treatment, TRASH-ASOs significantly reduced cell-growth in all tested cell-lines (Fig. 3a). Using clonogenic assays, we further investigated the colony growth potential in three melanoma celllines after TRASH-ASO treatment. TRASH-ASOs drastically reduced the capability of melanoma cells to produce colonies when compared to Control-ASO treatment (Fig. 3b). To test if these inhibitory effects of TRASH-ASO treatment may be partially related to the TRASH-hnRNPA2/B1 axis, we performed hnRNPA2/B1-ASO treatment in the D04 cell-line. Like TRASH-ASO treatment, hnRNPA2/B1-ASO 
treatment also significantly reduced cell-growth, but to a lesser extent. (Fig. 3b-c). Afterwards, we investigated if the observed ASO treatment effects are due to apoptotic cell death. As a readout for the induction of apoptosis, caspase-3 \& -7 activity was significantly increased 3-fold after TRASH-, and 1.7fold after hnRNPA2/B1-ASO treatment (Fig. 3d).

To examine the biomolecular changes upon TRASH-inhibition in melanoma, D04 cells were treated with TRASH-ASOs and Control-ASOs and RNA was extracted and used for RNA-Seq. Differential expression (DE) analysis showed that TRASH-ASOs had a global effect on gene expression. We found that 574 genes were down-regulated, and 493 genes were up-regulated, when compared to Control-ASO treatment (Cut off was $>1.5$-fold change and FDR $<0.05$, Suppl. Table 1). GO term analysis revealed that the top enriched GO term clusters associated with the down-regulated genes were related to "ECM-receptor interaction" and "PI3K-AKT signaling pathway", while the top enriched GO term clusters associated with the up-regulated genes included the terms "protein tyrosine kinase activity" (GO: 0004713) and "Ras guanyl-nucleotide exchange factor activity" (GO0005088) (Suppl. Table 2). These GO terms consisted of genes encoding growth factors, tyrosine kinases, G protein coupled receptor subunits, and collagen subunits.

These findings suggest that TRASH governs melanoma cell survival and inhibits apoptosis to a stronger extent than its protein binding partner hnRNPA2/B1 and that TRASH may execute its anti-apoptotic functions as a regulator of the MAPK and PI3K-AKT signaling cascade.

\section{$\underline{\text { Kinase activity profiling reveals unique anti-apoptotic features of TRASH-expression }}$}

Kinases cover a wide range of apoptosis regulating functions in cancer. Given the findings that TRASHASO treatment strongly affects the transcriptional regulation of genes that are related to kinase signaling pathways, we aimed to perform functional profiling of kinase activity shifts triggered by TRASHinhibition. To do so, we used a kinase activity screening platform ${ }^{35}$ (named High Throughput Kinase 
Activity Mapping - HT-KAM) that enables the simultaneous identification of kinase enzymes functional state in cancer cells across a broad range of kinase families (see Methods for details). We generated protein extracts of two versions of the D04 (D04 - treatment naïve; D04RM - trametinib resistant) and the MM415 melanoma cell-lines, treated with Control-ASOs or TRASH-ASOs. We tested these cell extracts on HT-KAM and performed unsupervised hierarchical clustering of peptide-associated phosphorylation profiles (Fig. 4a) and of kinase activity signatures (Fig 4b). The changes in kinases' activity upon TRASH-ASO treatment indicate conserved responses across cell-lines, whether kinases are up-regulated or down-regulated (respectively in yellow or blue in Fig. 4b).

Due to the effects of TRASH-ASO treatment on cell viability and apoptosis induction (Fig. 3a+d), we focused on kinases with anti-apoptotic functions. We found that the pro-survival/proto-oncogenic kinases AKT1, CDK1, LYN, YES1, CHEK1, PKCA, STK11, PKCa and PIM1 were significantly less active upon TRASH-inhibition (Fig. 4c left panel). These kinases have been reported to regulate the state of caspases and pro-survival pathways including the RAF-MAPK and PI3K-AKT axes. ${ }^{37-44}$

To further test if these observations are TRASH-ASO treatment specific, we generated MALAT1-ASO treated extracts from the same cell-line models. MALAT1 is a known oncogenic IncRNA in various types of cancer, including melanoma. ${ }^{45,46}$ MALAT1-ASO treatment reduced cell-growth but displayed a significantly reduced effect on apoptosis induction in comparison to TRASH-inhibition $(p=0.002$ for 1.5 fold versus 3.0-fold Caspase-3 \& -7 activity increase respectively in Fig. 4d and Fig. 3d). Using the HTKAM platform, we found that the activity of the kinases associated with cell-survival were not downregulated in MALAT1-ASO treated cells (Fig. 4c right panel), but significantly and specifically downregulated upon TRASH-ASO treatment (Fig. 4c, p < 0.00007; Fig. 4e, kinase signatures of TRASH-, versus MALAT1-ASO treatment). In summary, our data indicate that TRASH-ASO treatment specifically downregulates the activity of anti-apoptotic kinases and pro-survival signaling pathways in melanoma cells, supporting the potential therapeutic relevance of TRASH-ASO treatment (Fig. 4f). 
$\underline{\text { TRASH-ASO treatment shows characteristics of potential clinical value }}$

The MEK-inhibitor (MEKi) trametinib is a FDA approved drug for the treatment of melanoma as monoand combinatorial therapy and used in clinics worldwide. ${ }^{47}$ Drug resistance is the main limiting factor in modern oncology. ${ }^{48}$ Therefore, therapeutic applications that reduce growth of drug resistant tumors are urgently needed. TRASH-ASO treatment in a panel of cell-lines that are resistant to MEKi led to significant cell-growth reduction, comparable to the effect seen in their non-resistant treatment-naïve cell-line counterparts (Fig. 3a and 5a). Combinational application of drugs is a common strategy in clinical oncology to synergize drug effects and to hamper the development of drug resistance. ${ }^{49,50} \mathrm{We}$ show synergistic effects between TRASH-ASOs and MEKi in a broad panel of different concentrations and combinations in the melanoma cell-line D04 and in the primary patient-derived cell-line AV5. Synergy strongly increased with higher concentrations of TRASH-ASOs. More importantly, no notable effects of drug antagonism in regards of one drug blocking the effectiveness of the other drug, could be observed (Fig. 5b).

Next, we rescued cells that survived initial TRASH-ASO and MEKi treatment and after a phase of regrowth in drug free media, we repeated the preceding drug treatment. D04 cells responded with increased vulnerability to TRASH-ASO treatment, implying that no drug resistance could be measured. On the other hand, D04 cells that underwent MEKi responded with significantly less cell-growth inhibition to further MEKi, implying that these cells developed resistance mechanisms that decreased vulnerability to MEKi (Fig. 5c). To further evaluate the clinical potential of TRASH-inhibition in melanoma, we aimed to test the effects of TRASH-ASO treatment versus Control-ASO treatment in mouse-models of melanoma. We treated xenograft mouse-models harboring melanoma cell-line tumors (D04), primary melanoma cell tumors (AV5) and a patient-derived tumor xenograft (PDX). Systemic treatment with $60 \mu \mathrm{g}$ subcutaneous ASO injections twice a week for 21 days, co-applied with an in vivo transfection reagent, significantly reduced tumor growth in the TRASH-ASO treatment groups in all three 
mouse models (Fig. 5d). The PDX tumor model TM01341 showed extremely high rates of tumor growth. Tumor growth could be significantly hampered in the TRASH-ASO treatment group, but in accordance with institutional guidelines for maximum acceptable tumor sizes, Control-ASO group mice had to be euthanized before the desired endpoint of the experiment. To simulate the experiment to the desired endpoint, tumor growth in the PDX control group was forecasted using a regression model (Fig. 5d). There were no significant differences in mice weight change between the TRASH-ASO and Control-ASO treatment group in any of the three xenograft tumor models (Fig. 5d). qRT-PCR of tumor tissue extracted after the conclusion of the treatment period showed that in vivo TRASH-ASO treatment strongly reduced TRASH-expression in D04 tumors (Fig. 5e). Under certain conditions ASO treatment can lead to toxic side effects, in particular hepatotoxicity. ${ }^{51}$ To investigate potential toxicity, liver tissue of treated mice was collected for $\mathrm{H}+\mathrm{E}$ staining after the end of the treatment period. Neither animals receiving TRASH-ASOs nor those receiving Control-ASOs had any detectable pathologic changes in liver tissue (Fig. 5f). Additionally, immunohistochemistry staining for the apoptosis marker cleaved-caspase-3 confirmed induction of apoptosis through systemic TRASH-ASO in vivo treatment compared to ControlASO treatment in D04 tumors (Fig. 5f).

In summary, these findings show that TRASH-ASOs could help to bypass the resistance of melanoma cells to MEKi therapy. Additionally, TRASH-ASO treatment has the potential to amplify the efficiency of MEKi. Mechanisms of resistance to RNAi have been reported in mammalian cells. ${ }^{52}$ However, to our knowledge, no data regarding resistance mechanisms against ASO mediated IncRNA depleting therapy in mammalian cells exists. Our findings highlight that in contrast to MEKi, no early-onset treatment resistance could be observed for TRASH-ASO treatment in melanoma. Most notably, systemic TRASHASO treatment induces apoptosis and significantly reduces TRASH-expression and tumor growth in vivo while showing no signs of toxicity. 


\section{Discussion}

There has been major progress in the development of melanoma therapeutics in the past decade, but many patients have limited benefit from these advances due to the acquisition of resistance to treatment. Therefore, additional treatment options are urgently needed. Over $40 \%$ of human cancers are caused and characterized by MAPK-pathway hyper-activation, which propels expression of essential oncogenic regulators that can serve as therapeutic targets in cancer therapy. ${ }^{2,11,53}$ Using a novel pipeline that is composed of a broad set of analytical in silico and in vitro steps, we identified a set of IncRNAs that are up-regulated through MAPK activation, including the nuclear enriched IncRNA TRASH.

Previous reports indicate that TRASH could play a role in non-alcoholic fatty liver disease, but to our knowledge, its oncogenic features have never been unveiled before. ${ }^{54}$ Our findings indicate that TRASHexpression is an essential event for melanoma cell-survival. With the ultimate goal of clinical applicability in mind, we used improved ASOs to inhibit TRASH-expression. Systemic in vivo TRASH-ASO treatment greatly reduced tumor growth through induction of apoptotic cell death in cell-line and patient-derived tumor xenograft melanoma mouse models, while showing no signs of hepatotoxicity or treatment related weight loss. Furthermore, TRASH-ASO treatment strongly reduced cell-growth and colony formation in vitro in a broad range of melanoma cell-lines and primary human melanoma cells, while having no such effects on non-malignant melanocytic cell-lines. Some of the oncogenic features of TRASH may rely on the stabilizing effect on its protein-binding partner, the product of the anti-apoptotic oncogene hnRNPA2/B1. Analyses of patient-derived melanoma and non-malignant skin tissues from TCGA and GTEx databases demonstrate that TRASH and hnRNPA2/B1 up-regulation is tightly correlated in melanoma. Given the melanoma cell-growth reducing effects of hnRNPA2/B1-ASOs in vitro, we propose that the functional axis of TRASH and hnRNPA2/B1 contributes to the oncogenic nature of TRASH and is a critical mechanism for melanoma cell homeostasis. 
TRASH-ASO treatment followed by transcriptome analysis and GO term analysis provided additional indication that TRASH knockdown may down-regulate the pro-survival MAPK and PI3K-AKT signaling pathways.

ASOs that down-regulate transcription of apoptosis inhibiting coding genes have already been proven to lead to long remission rates in cancer in clinical trials. ${ }^{18}$ Our findings indicate that TRASH is a novel and central IncRNA that can be targeted for apoptosis-inhibition. TRASH-ASO treatment induced apoptosis through specific down-regulation of the activity of the anti-apoptotic/pro-survival kinases AKT1, CDK1, LYN, YES1, CHEK1, PKCA, STK11, PKCa and PIM1. Kinases of the AKT family and CHEK1 inhibit caspase activity. Lyn is a caspase-activation dependent regulator of AKT. ${ }^{37-39,41}$ PKCa stimulates AKT-dependent RAF-1-activation. ${ }^{40}$ Yes1-inhibition has been reported to increase apoptotic effects of chemotherapy and it is positively regulated by the kinase CDK1. ${ }^{43}$ Tumor cells lacking the upstream master kinase STK11 are hypersensitive to apoptosis. ${ }^{42}$ PIM1 regulates cell-survival through down-regulation of the MAPKpathway kinase ASK1. ${ }^{44}$ An increasing number of IncRNAs playing crucial and specific roles in cancer have been identified, with MALAT1 being one of the well-known examples. ${ }^{17}$ Comparing the effects on kinase activity profiles of TRASH-, and MALAT1-inhibition demonstrated the unique pro-survival and anti-apoptotic features of TRASH. To our knowledge, such a pattern of anti-apoptotic kinase activity inhibition through ASO mediated gene silencing has never been reported.

Our research identified additional clinically significant features of TRASH-ASO treatment: MEKiresistance does not desensitize melanoma cells to their TRASH-dependency in vitro, indicating that TRASH-ASOs presents the prerequisite to serve as treatment for melanoma refractory to MEKi. Dual application of TRASH-ASOs and MEKi amplifies the effects of mono application in vitro, demonstrating the synergistic effects of multi-drug regimens that clinical dermato-oncologists strive for. In addition, melanoma cells display early-onset drug resistance to MEKi treatment, while no such response was observed with TRASH-ASO treatment. 
For systemic in vivo application, we combined TRASH-ASOs with the transfection reagent JetPEI ${ }^{\circledR}$, which has been tested in clinical trials, including melanoma gene therapy. ${ }^{55-58}$ Interestingly, the combination of TRASH-ASOs and JetPEI ${ }^{\circledR}$ achieved tumor growth reduction using 10-20x reduced ASO dosage, when compared to other ASO treatment studies in rodents..$^{59,60}$

ASO based therapy has gained approval by the Food and Drug Administration (FDA) and the European Medicines Agency (EMA) and is currently tested in clinical trials for a large repertoire of diseases and with various application routes. ${ }^{19}$ Therapeutic options that aim to lower the threshold for apoptosis are a central strategy in oncology and IncRNA-targeting therapy showed promising results in cancer treatment. ${ }^{17}$ Hence, TRASH-ASO treatment could play an important role in RNA-targeting melanoma therapy and even be beneficiary for patients who suffer from other malignancies. 


\section{Methods}

Bioinformatic pipeline for identifying MAPK-responsive IncRNAs

\section{Reference Annotation}

A custom reference annotation of total 75,506 transcripts, referring to 35,101 genes, of which 16,405

were classified as non-coding, was built by integrating 13,870 IncRNA genes from the GENCODE ${ }^{61}$ (V19, July 2013 freeze, GRCh37, downloaded March 2015) into the RefSeq ${ }^{62}$ database (release 57, downloaded March 2013). Cuffcompare (version 2.1.1) was used to cut out redundant transcripts.

\section{Assembly and identification of previously unidentified IncRNAs}

After alignment to the human genome with TopHat (version 2.0.11), the reads were assembled into transcripts with Cufflinks (version 2.1.1). To discover novel IncRNAs, we excluded all transcript IDs that overlapped with any gene IDs from our initial reference annotation. To filter out transcriptional noise, we kept only multi-exonic transcript IDs which were $>200 \mathrm{bp}$ and had at least one intron region > $10 \mathrm{bp}$. Next, isoforms were merged with Cuffcompare.

\section{Coding Potential Assessment of Transcripts}

To identify transcript IDs with a coding potential, we ran (a) the HMMER3 algorithm (considering all 6 open reading frames) to identify any protein family domain as noted in the Pfam database (release 27.0, Pfam-A and Pfam-B domains considered) and (b) the Coding Potential Assessment Tool (CPAT v1.2.1).

\section{Filter for DE genes}

Cuffdiff (v.2.1.1) was used to identify differential gene expression analysis between $\mathrm{PHM}^{\mathrm{E}}$ and $\mathrm{PHM}^{\mathrm{Q}}{ }^{61}$. From a reference of 35,905 genes, we discarded genes with FPKM $<0.2$ in both conditions and kept genes with log2-fold change $>1$ or $<-1$. Cufflinks was used to obtain FPKM-values in RNA-Seq Data from the D04 and MM415 melanoma cell-lines. Log2 transformations were performed to calculate expression fold change in the comparisons: 1) PHM ${ }^{\mathrm{E}}$ vs. PHMQ61, 2) PHM vs. D04, 3) PHM vs. MM415. Genes that 
had a log 2 -fold change $>1$ or $<-1$ and showed same tendency in all three comparisons were considered as differentially expressed.

\section{TCGA data extraction and processing}

Raw .fastq files were obtained from The Cancer Genome Atlas (TCGA) from 86 NRAS mutant melanoma patients from the TCGA-SKCM dataset. Transcript de-novo assembly was performed as described above.

\section{Animal models}

Rodent experimental procedures were approved by the Office of Research institutional Animal Care and Use Program (IACUC) at the University of San Francisco (UCSF). All in vivo studies were conducted under the authorized protocol number AN174613-03. Mice were maintained in a pathogen free environment and had free access to food and water. For PDX tumor models, the PDX type TM01341, derived from liver metastasis of a male melanoma patient, was engrafted on 4- to 6-week-old NOD.Cg-Prkdc ${ }^{\text {scid }}$ $\| 2 \mathrm{rg}^{\mathrm{tm} 1 \mathrm{Wjl}} / \mathrm{SzJ}$ mice (Stock.no 005557) on the right posterior dorsal flank ( $\mathrm{n}=4 /$ group). For cell-line models, $2 \times 10^{\wedge}{ }^{6}$ D04 ( $n=5 /$ group) and AV5 ( $n=3 /$ group) cells in $150 \mu$ l of PBS and 50 $\mu$ l of Matrigel were subcutaneously injected on the right posterior dorsal flank of 4- to 6-week-old homozygous nude Foxn $1^{\text {nu }} /$ Foxn $1^{\text {nu }}$ mice (Stock.no 007850). Mice and PDX tissue were obtained from JAX ${ }^{\circledR}$. Tumor size was measured using a digital caliper and the formula $0.5 \times$ (length $\times($ width^2)) was used to calculate tumor

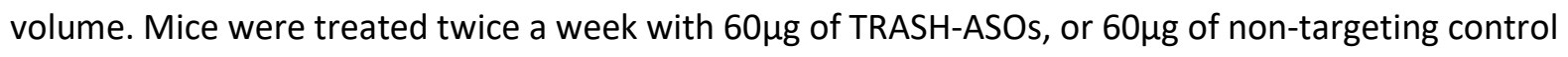
ASOs and $9.6 \mu \mathrm{l}$ of in vivo JetPEI ${ }^{\circledR}$ diluted in an overall amount of $200 \mu \mathrm{l} 5 \%$ glucose. ASO injections were applied subcutaneously. Mice were weighted twice a week and observed for signs of distress or disorder. Mice were euthanized after three weeks of ASO application or when tumors reached a diameter of $>2 \mathrm{~cm}$. All experiments were performed in accordance with the UCSF Laboratory Animal Resource Center (LARC) guidelines. After euthanasia, tumor samples and liver tissue were excised and fixed in formalin solution, followed by storing in $70 \%$ ethanol and Immunohistochemistry staining. Tumor samples were also placed in RNAlater ${ }^{\mathrm{TM}}$ Stabilization Solution (Thermo Fisher Scientific ${ }^{\circledR}$ ) and 
stored at $-20^{\circ} \mathrm{C}$. Invitrogen ${ }^{\mathrm{TM}} \mathrm{TRIzol}^{\mathrm{TM}}$ Solution (Thermo Fisher Scientific ${ }^{\circledast}$ ) was used to extract RNA from tissue and qPCR was performed to analyze gene expression.

\section{Immmunohistochemistry}

Tumor tissues were extracted from mice immediately after euthanasia and fixed in $10 \%$ neutral buffered formalin for 24 hours, followed by storage in $70 \%$ EtOH. Histopathology was conducted by the UCSF Histology and Biomarker Core. FFPE sections were collected at 4-micron thickness and mounted on center of positively charged glass slides (Superfrost Plus Slides Fisher Item\# 12-550-15) following lab standard procedures for the sectioning of paraffin blocks. Slides where air-dried overnight for 8 to 24 hours then baked and deparaffinized. For hematoxylin and eosin (H\&E) staining, slides were baked at $60^{\circ} \mathrm{C}$ for 15 minutes prior to staining; for immunohistochemistry (IHC) staining, slides were baked overnight at $60^{\circ} \mathrm{C}$ prior to staining. H\&E staining was performed on the Leica XL Autostainer using hematoxylin (part no. 6765015 from Thermo Scientific) for seven minutes and alcoholic eosin (Part No. 6765040 from Thermo Scientific) for 20 seconds. IHC was done on the Roche Ventana Discovery Ultra Autostainer using antibodies for Cleaved-Caspase-3 (Cell Signaling Technology ${ }^{\circledR}$, cat.no.: 9664S, dilution 1:600). Ventana Protocol Summary: CC1 cell conditioning at $95^{\circ} \mathrm{C}$ for 24 minutes, Inhibitor time 12 minutes, primary antibody incubation at $36^{\circ} \mathrm{C}$ for 60 minutes, anti-Rabbit $\mathrm{HQ}$ detection at $37^{\circ} \mathrm{C}$ for 12 minutes, Chromagen: DAB. The Zeiss Axio scanner was used for digital slide scanning with brightfield images collected at 20X magnification using Zeiss Zen software.

\section{Cell culture}

Human melanoma cell-line VMM39 (NRAS-Mutation: Q61L, Q61R) was purchased from American Type Culture Collection (ATCC ${ }^{\circledR}$ ). Human melanoma cell-lines D04 (NRAS-Mutation: Q61L), MM415 (NRASMutation: Q61L), WM1366 (NRAS-Mutation: Q61L), WM3629 (BRAF-Mutation D594G, NRAS-Mutation: G12D), WM3211 (c-Kit-Mutation: L576P), Sk-Mel-2 (NRAS-Mutation: Q61R) and Sk-Mel-28 (BRAFMutation V600E) were gifted by Dr. Boris Bastian at the UCSF. Primary human melanoma cell-line 
Hs852.T (NRAS-Mutation: G12V) was purchased from the Cell and Genome Engineering Core (CGEC) at the UCSF. Primary human melanoma cell-line AV5 (BRAF-Mutation: V600E) was obtained from metastasis of a melanoma patient. All experimental protocols were approved by UCSF Human Research Protection Program Institutional Review Board (IRB\# 12-0948). Methods were carried out in accordance with relevant guidelines and regulations. Resistant cell-lines D04RM, MM415RM, Sk-Mel-2RM and WM3629RM were established as previously described. ${ }^{16}$ Primary human melanocytic cell-lines (PHM) from infant foreskin of five healthy donors were available in our cell repository. Melanoma cell-lines were maintained in RPMI 1640 media supplemented with 10\% (vol/vol) heat inactivated fetal bovine serum. Melanocytes were maintained in M254 medium with HMGS supplements (1x final solution). All cell-lines were incubated at $37^{\circ} \mathrm{C}$ under $5 \% \mathrm{CO} 2$.

\section{Viral transduction}

NRAS $^{\mathrm{Q} 61 R}$ cDNA was cloned into the Gateway entry vector pENTR/D-topo. pENTR/D-topo-NRAS ${ }^{\mathrm{Q} 61 \mathrm{R}}$ was subjected to site-directed mutagenesis to generate mutants which were then validated by Sangersequencing. NRAS ${ }^{061 R}$ CDNA in pENTR was cloned into the Gateway cloning-enabled destination vector gFG12. After lentiviral transduction, cells were grown for two weeks followed by cell sorting facilitated by GFP expression intensity on a FACS Aria II cell sorter.

\section{Sanger-Sequencing}

RNA from PHM cell-lines was extracted using Purelink ${ }^{\top M}$ RNA extraction kit (Thermo Fisher Scientific ${ }^{\circledR}$ ) and transcribed into cDNA. Sanger-Sequencing was performed by Quintarabio Inc. For NRAS amplification, the forward primer CGCACTGACAATCCAGCTAA and the reverse primer TCGCCTGTCCTCATGTATTG were used.

\section{Protein extraction and immunoblotting}

$1 \times 10^{\wedge 5}$ D04 cells were seeded in six well-plates one day prior to transfection. One day after seeding, cells were incubated in media with final oligonucleotide concentration. Total protein lysates were 
homogenized in 1x RIPA buffer and Halt protease and phosphatase inhibitor cocktail (Thermo Fisher Scientific ${ }^{\circledR}$ ) followed by centrifugation at $14,000 \mathrm{RPM} /$ minute at $4^{\circ} \mathrm{C}$. Protein concentration was quantified using the Pierce ${ }^{\mathrm{TM}}$ BCA Assay Kit (ThermoFisher Scientific ${ }^{\circledR}$ ). Linear absorbance was measured using the Synergy ${ }^{\mathrm{TM}}$ HT (Agilent Technologies Inc) plate reader. Total protein in $1 \times$ Laemmli buffer with 10\% 2- mercaptoethanol was separated by SDS/PAGE, transferred for $15 \mathrm{~h}$ to a PVDF membrane (IPVH00010; MilliporeSigma ${ }^{\circledR}$ ) by electroblotting with 20\% (vol/vol) methanol, and blocked for $1 \mathrm{~h}$ in in Intercept (TBS) blocking buffer (LI-COR $\left.{ }^{\circledR}\right)$. Membranes were incubated overnight at $4{ }^{\circ} \mathrm{C}$ with primary antiserum for hnRNPA2/B1 (Abcam ${ }^{\circledR}$, cat.no.: ab31645, dilution 1:750) and $\beta$-Actin (Cell Signaling Technology ${ }^{\circledast}$, cat.no.: 8457, dilution 1:2500) following incubation with secondary Goat Anti-Rabbit serum (LI-COR ${ }^{\circledR}$, dilution 1:5000) for $1 \mathrm{~h}$ and scanned using the Li-COR $^{\circledR}$ Odyssey ${ }^{\circledR}$ Imaging system.

\section{RNA extraction and quantitative real-time PCR (qRT-PCR)}

TRIzol $^{\text {TM }}$ Solution (Thermo Fisher Scientific ${ }^{\circledR}$ ), Phenol:chloroform:isoamyl alcohol (MilliporeSigma ${ }^{\circledR}$ ) or NucleoSpin ${ }^{\circledR}$ RNA kit (Takara Bio USA, Inc.) were used for extracting Total RNA from cells and tissues according to the manufacturer's instructions. Total RNA was quantified by NanoDrop ${ }^{\text {TM ND-1000 }}$ (Thermo Fisher Scientific ${ }^{\circledR}$ ) or Quibit ${ }^{\text {TM }} 4$ (Thermo Fisher Scientific ${ }^{\circledR}$ ). 50ng or RNA was reverse transcribed using the cDNA synthesis and gDNA removal QuantiTect ${ }^{\circledR}$ Reverse Transcription Kit (Thermo Fisher Scientific ${ }^{\circledR}$ ). Real time PCR was performed using the $\mathrm{iTaq}^{\mathrm{TM}}$ Universal SYBR ${ }^{\circledR}$ Green Supermix (Bio-

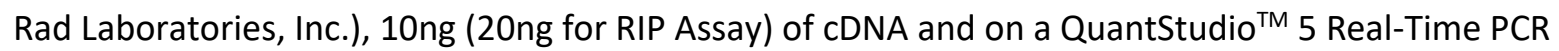
System or a 7500 fast real time PCR system (both from Thermo Fisher Scientific ${ }^{\circledR}$ ). Relative gene expression was calculated using the comparative $\mathrm{Ct}$ method, normalized to GAPDH or $\beta$-actin. Primers are listed in Supplementary Table 3. TRASH primer pair 4 was used for knockdown evaluation, splicing efficiency evaluation and RIP. TRASH primer pairs 1, 2, and 3 were used for splicing efficiency evaluation. Primers were obtained from Integrated DNA Technologies, Inc.

\section{Purification of Nuclear and Cytoplasmic RNA}


Total nuclear and cytoplasmic extracts were obtained using the SurePrep ${ }^{\mathrm{TM}}$ Nuclear/Cytoplasmic RNA purification kit (Thermo Fisher Scientific ${ }^{\circledR}$ ) according to the manufacturer's instructions. RNA extraction and qRT-PCR were performed as described above using TRASH primer pair 4, GAPDH primer pair forward (TGGAAGGACTCATGACCACA) and reverse (GCCATCACGCCACAGTTT), MALAT1 primer pair forward (GACGGAGGTTGAGATGAAGC) and reverse (ATTCGGGGCTCTGTAGTCCT), H19 primer pair forward (ATCGGTGCCTCAGCGTTCGG) and reverse (CTGTCCTCGCCGTCACACCG) and NEAT1 primer pair forward (СTTCCTCCCTTTAACTTATCCATTCAC) and reverse (СTCTTCCTCCACCATTACCAACAATAC).

\section{Oligonucleotide transfection}

EsiRNA was generated following standard protocol. ${ }^{63}$ Primer sequences

TCACTATAGGGAGAGACACTCAAAGCCTGAGTAACAGA and

TCACTATAGGGAGACTGACTGAGATTTTATTGAGCTGTG were used to create TRASH-targeting esiRNA.

SiRNA was purchased from Horizon Discovery Biosciences Ltd and designed using the siDESIGN software. For TRASH-targeting siRNA design, the sequence ACAAAGAGAGACAGGAAAUUU was used. For pooled non-targeting control siRNA design, the sequences UGGUUUACAUGUCGACUAA, UGGUUUACAUGUUGUGUGA, UGGUUUACAUGUUUUCUGA and UGGUUUACAUGUUUUCCUA were used. ASO-GapmeRs were purchased from QIAGEN N.V. and designed using the GeneGlobe design and analysis hub. For TRASH-targeting ASO design, the sequence GACTGGAGATAATTAA was used for in vitro experiments and TGCGCGGCGGAAAGAA for in vivo. For hnRNPA2/B1-targeting ASO design, the sequence GACCGTAGTTAGAGG was used. For non-targeting control ASO design, the QIAGEN N.V. standard sequence AACACGTCTATACGC was used. Final concentrations of esiRNA, siRNA and ASOs in media are listed in figure legends and for in vitro experiments, the transfection reagent Lipofectamine $\mathrm{T}^{\mathrm{TM}}$ $3000(2 \mathrm{ul} / \mathrm{ml})$ was added according to the manufacturer's instructions.

\section{Expression analysis in TCGA and GTEx}


The analysis of TCGA/GTEx gene expression data was done in R. For TCGA data, the SKCM dataset ( $n=469)$ was used. The GDCquery function of the TCGAbiolinks package was run with the following parameters: project ="TCGA-SKCM", data.category ="Transcriptome Profiling", data.type ="Gene Expression Quantification", workflow.type =“HTSeq - FPKM". GDCdownload and GDCprepare then produce a RangedSummarizedExperiment. Expression values are then stored in a data frame and converted to TPM by dividing each FPKM-value by the total FPKM of each sample and multiplying by 10^6. To retrieve GTEx data (n=394), “GTEx_Analysis_2017-06-05_v8_RNASeQCv1.1.9_gene_tpm.gct.gz" was downloaded from gtexportal.org/home/datasets. ${ }^{64}$ Skin samples within the GTEx dataset were identified by referencing https://www.ebi.ac.uk/arrayexpress/experiments/E-MTAB5214/samples/?s_page=59\&s_pagesize $=500 \&$ s_sortby=col_8\&s_sortorder=ascending. The raw read counts were converted to TPM-values and then transformed to $\log 2$ scale. A value of 1 was added to avoid taking log of zero. For both TCGA and GTEx, duplicate genes were removed. If a patient provided multiple specimens, only the first would be used. The ensemble ID for our genes of interest were ENSG00000225792 (TRASH) and ENSG00000122566 (hnRNP2/B1). Cor.test was applied to find the correlation between each gene and TRASH, and the same for hnRNPA2/B1. Spearman's correlation coefficient $(\rho)$ was used to measure rank correlation. 2000 random genes were sampled from both TCGA and GTEx datasets. The correlation of TRASH and hnRNPA2/B1 was ranked against 200 random gene correlations with TRASH and hnRNPA2/B1 each for 10 iterations.

\section{Cell viability assay}

Dependent on cell doubling time, $0.7-2 \times 10^{\wedge 3}$ cells were seeded in 96 well-plates one day prior to transfection. One day after seeding, cells were incubated in media with final oligonucleotide concentration and/or MEKi and transfection reagent. Three (synergy experiments) or five (solely ASOs) days after transfection Total luminescence was measured on the Synergy ${ }^{\mathrm{TM}}$ HT (Agilent Technologies Inc) 
plate reader using Promega ${ }^{\circledR}$ CellTiter-Glo ${ }^{\circledR}$ and Gen5 software. Cell-growth is shown in relation to cells incubated with Control-ASOs.

\section{Calculation of Combinational Index (CI)}

The effects of drug combinations on cell growth were assessed by calculating the combination index $(\mathrm{Cl})$ $=\log _{2}\left[E_{\mathrm{a}, \mathrm{b}} /\left(-E_{\mathrm{a}} \mathrm{X} E_{\mathrm{b}}\right)\right]$, where $E_{\mathrm{a}}$ and $E_{\mathrm{b}}$ correspond to the effects of drugs $\mathrm{A}$ and $\mathrm{B}$ alone at a given concentration, and $E$ a,b corresponds to the combined effects of drugs $A$ and $B$ at the same concentration, and a combination index of $<0$ indicates synergy while a combination index of $>0$ relates to an antagonistic effect. The individual combination indices per drug combination were then averaged.

\section{Caspase Glo 3 \& 7 assay}

Dependent on cell doubling time, $2-3 \times 10^{\wedge 3}$ cells were seeded in 96 well-plates one day prior to transfection. One day after transfection Total luminescence was measured on the the Synergy ${ }^{\mathrm{TM}}$ HT (Agilent Technologies Inc) plate reader using The Promega ${ }^{\circledR}$ Caspase-Glo ${ }^{\circledR}$ 3/7 Assay and Gen5 software. Experiments were performed in quadruplicates.

\section{RNA-Binding Protein Immunoprecipitation}

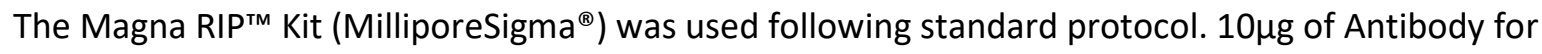
Rabbit IgG (MilliporeSigma ${ }^{\circledR}$, Cat.no.: PP64B) and hnRNPA2/B1 (Proteintech ${ }^{\circledR}$, Cat.no.: 14813-1-AP) were used to load magnetic beads. RNA precipitate was subjected to qRT-qPCR analysis.

\section{Colony formation Assay}

Dependent on cell doubling time, $1-2 \times 10^{\wedge 3}$ cells were seeded in six well-plates. One day after seeding, cells were incubated in media with 50nM oligonucleotide concentration and transfection reagent. Six days after transfection, cells were washed with PBS, fixed with $10 \%$ neutral buffered formalin, and stained with $0.1 \%$ crystal violet solution. Colonies were defined as cell conglomerates with $>50$ cells. Digital images of plates were evaluated by two independent reviewers for colony counts. The final counts were calculated as the average count of both reviewers for all triplicates. 


\section{Kinase activity mapping technology}

For samples to be analyzed with the HT-KAM platform, cells were treated with ASOs for 24 hours and at 85\% confluency cells were washed three times with cold PBS and lysed with freshly prepared 1X cell lysis buffer ( $1 \mathrm{ml}$ per $2.5 \times 106$ cells) (10x Cell lysis buffer, Cell Signaling Technology ${ }^{\circledR}$, Cat.No. 9803), complemented with 1x Halt Protease and Phosphatase (Thermo Fisher Scientific ${ }^{\circledR}$ Cat.No 1861281). Lysates were scraped off, spun down at $14,000 \mathrm{rpm}$. at $4^{\circ} \mathrm{C}$ for $15 \mathrm{~min}$ and supernatants were stored at $-80^{\circ} \mathrm{C}$. The high throughput kinase activity mapping (HT-KAM) platform uses arrays of peptides that act as sensors of phosphorylation activity. ${ }^{35}$ The phospho-catalytic signature of samples is established from simultaneously occurring ATP-consumption tests measured in the presence of individual peptides that are experimentally isolated from each other. Assays were run in 384 well-plates, where each experimental well contains one peptide. The final $8 \mu \mathrm{L}$ reaction mixtures per well contain: (a) kinase assay buffer (1X KaB: $2.5 \mathrm{mM}$ Tris- $\mathrm{HCl}(\mathrm{pH} 7.5), 1 \mathrm{mM} \mathrm{MgCl}_{2}, 0.01 \mathrm{mM} \mathrm{Na}_{3} \mathrm{VO}_{4}, 0.5 \mathrm{mM}$ ?]glycerophosphate, 0.2mM dithiothreitol (DTT), prepared daily; (10X KaB Cell Signaling Technology ${ }^{\circledR}$, Cat.No. 9802), (b) 250nM ATP (prepared daily with 1X KaB; Cell Signaling Technology ${ }^{\circledR}$ Cat.No. 9804), (c) $200 \mu \mathrm{g} / \mathrm{ml}$ 11-mer peptide (lyophilized stocks originally prepared as $1 \mathrm{mg} / \mathrm{ml}$ in $1 \mathrm{X} \mathrm{KaB}, 5 \% \mathrm{DMSO}$ ), and (d) samples made from cell at $\sim 10 \mu \mathrm{g} / \mathrm{ml}$ total protein extract. Samples are kept on ice and diluted in $1 \mathrm{X}$ $\mathrm{KaB}<30 \mathrm{~min}$ before being used. Controls with no-ATP, or no-peptide, or no-sample as well as ATP standards are run side-by-side within each 384 well-plate. High-throughput liquid dispensing of all reagents is performed using a Biomek ${ }^{\circledR}$ FX Laboratory Automation Workstation from Beckman Coulter. All reagents are kept on ice and plates on cold blocks until enzymatic reactions are started. Once the dispensing of the reaction mixtures is complete, the plates are incubated for $1 \mathrm{~h}$ at $30^{\circ} \mathrm{C}$. ATP is detected using Kinase-Glo revealing reagent (Promega ${ }^{\circledR}$; Cat.No V3772), which stops the activity of the kinases and produces a luminescent signal that directly correlates with the amount of remaining ATP in the samples. Luminescence is acquired using the Synergy 2 Multi-Mode Microplate Reader from BioTek. 
Luminescence data are inversely correlated with the amount of kinase activity. For a more detailed description of the peptide sensors design, sequence and connectivity between peptides and kinases, as well as data normalization steps and analysis, refer to: ${ }^{35,65,66}$. The activity of kinase enzymes is derived from their respective subset of biological peptide targets included in the assay.

\section{RNA-sequencing}

Total RNA was isolated using the RNeasy ${ }^{\circledR}$ mini-Kit (QIAGEN N.V.) following the manufacturer's protocol. Quality check for extracted RNA was done using 2100 Bioanalyzer (Agilent Technologies Inc.) or Tapestation 4200 System (Agilent Technologies Inc.). All samples had a RIN score $>8$. For samples used for identification of MAPK-responsive IncRNAs, cDNA-sequencing libraries were prepared using the Illumina ${ }^{\circledR}$ TruSeq $^{\circledR}$ Total RNA Sample kit and paired-end, 101-bp sequencing was performed by Centrillion Genomic Services (Centrillion Tech.) on an Illumina ${ }^{\circledR} \mathrm{HiSeq}^{\circledR}$ 2000. Sequence reads were aligned to the human genome (hg19) using TopHat (Version 2.0.11). For DE gene analysis of ASOtransfected D04 samples, NEBNext ${ }^{\circledR}$ ultra-RNA library prep kit (New England Biolabs ${ }^{\circledR}$ Inc.) with rRNA depletion and paired-end, 2x150-bp sequencing was performed by Genewiz ${ }^{\circledR}$ on an Illumina ${ }^{\circledR}$ HiSeq $^{\circledR}$ 4000. Sequence reads were aligned to the GRCh38 reference genome.

\section{Analysis of TRASH-ASO induced DE gene expression}

Differential expression (DE) analysis was done using DESeq2. Differentially expressed genes were defined by more than 1.5 -fold changes $\left(\log _{2}>0.58\right.$ or $<-0.58$ ) in expression with FDR $<0.05$. GO term analysis was done using DAVID ${ }^{67}$ Functional Annotation Clustering analysis (version 6.8). ${ }^{67,68}$

\section{Statistics and reproducibility}

Error bars in all the plots indicate mean \pm S.D. P-value $<0.05$ was considered statistically significant.

$* * * p$-value $<0.001, * * p$-value $<0.01, * p$-value $<0.05$ by one tailed Student's t-test. All experiments were performed at least three times, unless otherwise indicated. Statistics was calculated with Microsoft ${ }^{\circledR}$ Excel Version 2107. 


\section{Acknowledgements}

This work was supported by the American Skin Association, Verein zur Förderung der Dermatologischen Forschung (VFDF), Impact Melanoma and the Outrun the Sun, Inc. 2019 National Melanoma Research Scholar Award. Additionally, we thank William Chou at UCSF for technical support. Results are in part based upon data generated by the TCGA (https://www.cancer.gov/tcga) and GTEx Research Network: (https://gtexportal.org)

\section{Competing Interests Statement}

No potential competing interest was reported by the authors. 


\section{References}

1. Surveillance, Epidemiology, and End Results (SEER) Program (www.seer.cancer.gov) SEER*Stat Database: Populations - Total U.S. (1969-2019) <Katrina/Rita Adjustment> - Linked To County Attributes - Total U.S., 1969-2019 Counties, National Cancer Institute, DCCPS, Surveillance Research Program, released December 2020.

2. Yuan, J., Dong, X., Yap, J. \& Hu, J. The MAPK and AMPK signalings: interplay and implication in targeted cancer therapy. J Hematol Oncol 13, 113 (2020).

3. Attwood, M. M., Fabbro, D., Sokolov, A. V., Knapp, S. \& Schiöth, H. B. Trends in kinase drug discovery: targets, indications and inhibitor design. Nat Rev Drug Discov (2021) doi:10.1038/s41573-021-00252y.

4. Niessner, H. et al. BRAF Inhibitors Amplify the Proapoptotic Activity of MEK Inhibitors by Inducing ER Stress in NRAS-Mutant Melanoma. Clin Cancer Res 23, 6203-6214 (2017).

5. Carneiro, B. A. \& El-Deiry, W. S. Targeting apoptosis in cancer therapy. Nat Rev Clin Oncol 17, 395417 (2020).

6. Barceló, C. et al. Ribonucleoprotein HNRNPA2B1 Interacts With and Regulates Oncogenic KRAS in Pancreatic Ductal Adenocarcinoma Cells. Gastroenterology 147, 882-892.e8 (2014).

7. Chen, Z. et al. Integrative Analysis of NSCLC Identifies LINC01234 as an Oncogenic IncRNA that Interacts with HNRNPA2B1 and Regulates miR-106b Biogenesis. Molecular Therapy 28, 1479-1493 (2020).

8. Gupta, A. et al. The HNRNPA2B1-MST1R-Akt axis contributes to epithelial-to-mesenchymal transition in head and neck cancer. Lab Invest (2020) doi:10.1038/s41374-020-0466-8.

9. Wang, $\mathrm{H}$. et al. Long noncoding RNA miR503HG, a prognostic indicator, inhibits tumor metastasis by regulating the HNRNPA2B1/NF-KB pathway in hepatocellular carcinoma. Theranostics 8, 2814-2829 (2018). 
10. Shilo, A. et al. Splicing factor hnRNP A2 activates the Ras-MAPK-ERK pathway by controlling ARaf splicing in hepatocellular carcinoma development. RNA 20, 505-515 (2014).

11. Luke, J. J., Flaherty, K. T., Ribas, A. \& Long, G. V. Targeted agents and immunotherapies: optimizing outcomes in melanoma. Nat Rev Clin Oncol 14, 463-482 (2017).

12. Cabili, M. N. et al. Integrative annotation of human large intergenic noncoding RNAs reveals global properties and specific subclasses. Genes \& Development 25, 1915-1927 (2011).

13. Amodio, N. et al. MALAT1: a druggable long non-coding RNA for targeted anti-cancer approaches. J Hematol Oncol 11, 63 (2018).

14. Lin, A. et al. The LINK-A IncRNA interacts with PtdIns $(3,4,5) P 3$ to hyperactivate AKT and confer resistance to AKT inhibitors. Nat Cell Biol 19, 238-251 (2017).

15. Zhang, A. et al. LncRNA HOTAIR Enhances the Androgen-Receptor-Mediated Transcriptional Program and Drives Castration-Resistant Prostate Cancer. Cell Reports 13, 209-221 (2015).

16. Sanlorenzo, M. et al. The lincRNA MIRAT binds to IQGAP1 and modulates the MAPK pathway in NRAS mutant melanoma. Sci Rep 8, 10902 (2018).

17. Huarte, M. The emerging role of IncRNAs in cancer. Nat Med 21, 1253-1261 (2015).

18. Carter, B. Z. et al. XIAP antisense oligonucleotide (AEG35156) achieves target knockdown and induces apoptosis preferentially in CD34+38- cells in a phase $1 / 2$ study of patients with relapsed/refractory AML. Apoptosis 16, 67-74 (2011).

19. Dhuri, K. et al. Antisense Oligonucleotides: An Emerging Area in Drug Discovery and Development. JCM 9, 2004 (2020).

20. Winkle, M., El-Daly, S. M., Fabbri, M. \& Calin, G. A. Noncoding RNA therapeutics - challenges and potential solutions. Nat Rev Drug Discov 20, 629-651 (2021).

21. Fedorenko, I. V., Gibney, G. T. \& Smalley, K. S. M. NRAS mutant melanoma: biological behavior and future strategies for therapeutic management. Oncogene 32, 3009-3018 (2013). 
22. Platz, A., Egyhazi, S., Ringborg, U. \& Hansson, J. Human cutaneous melanoma; a review of NRAS and BRAF mutation frequencies in relation to histogenetic subclass and body site. Molecular Oncology 1, 395-405 (2008).

23. Posch, C. et al. Combined targeting of MEK and PI3K/mTOR effector pathways is necessary to effectively inhibit NRAS mutant melanoma in vitro and in vivo. Proceedings of the National Academy of Sciences 110, 4015-4020 (2013).

24. Posch, C. et al. Searching for the Chokehold of NRAS Mutant Melanoma. Journal of Investigative Dermatology 136, 1330-1336 (2016).

25. Pollock, P. M. et al. High frequency of BRAF mutations in nevi. Nat Genet 33, 19-20 (2003).

26. Karakas, D. \& Ozpolat, B. The Role of LncRNAs in Translation. Noncoding RNA 7, 16 (2021).

27. Statello, L., Guo, C.-J., Chen, L.-L. \& Huarte, M. Gene regulation by long non-coding RNAs and its biological functions. Nat Rev Mol Cell Biol 22, 96-118 (2021).

28. Singh, R. \& Valcárcel, J. Building specificity with nonspecific RNA-binding proteins. Nat Struct Mol Biol 12, 645-653 (2005).

29. Kole, R., Krainer, A. R. \& Altman, S. RNA therapeutics: beyond RNA interference and antisense oligonucleotides. Nat Rev Drug Discov 11, 125-140 (2012).

30. Lennox, K. A. \& Behlke, M. A. Cellular localization of long non-coding RNAs affects silencing by RNAi more than by antisense oligonucleotides. Nucleic Acids Res 44, 863-877 (2016).

31. Mathieson, T. et al. Systematic analysis of protein turnover in primary cells. Nat Commun 9, 689 (2018).

32. Liang, J. et al. The C-Kit Receptor-Mediated Signal Transduction and Tumor-Related Diseases. Int. J. Biol. Sci. 9, 435-443 (2013).

33. Vu, H. L. \& Aplin, A. E. Targeting mutant NRAS signaling pathways in melanoma. Pharmacological Research 107, 111-116 (2016). 
34. Dhomen, N. \& Marais, R. BRAF Signaling and Targeted Therapies in Melanoma.

Hematology/Oncology Clinics of North America 23, 529-545 (2009).

35. Coppé, J.-P. et al. Mapping phospho-catalytic dependencies of therapy-resistant tumours reveals actionable vulnerabilities. Nat. Cell Biol. 21, 778-790 (2019).

36. Vujic, l. et al. Metformin and trametinib have synergistic effects on cell viability and tumor growth in NRAS mutant cancer. Oncotarget 6, 969-978 (2015).

37. Luciano, F., Herrant, M., Jacquel, A., Ricci, J. \& Auberger, P. The P54-cleaved form of the tyrosine kinase Lyn generated by caspases during BCR-induced cell death in B lymphoma acts as a negative regulator of apoptosis. FASEB j. 17, 711-713 (2003).

38. Negro, R. et al. Overexpression of the autoimmunity-associated phosphatase PTPN22 promotes survival of antigen-stimulated CLL cells by selectively activating AKT. Blood 119, 6278-6287 (2012).

39. Zhong, D. et al. LKB1 Is Necessary for Akt-Mediated Phosphorylation of Proapoptotic Proteins. Cancer Res 68, 7270-7277 (2008).

40. Gutcher, I., Webb, P. R. \& Anderson, N. G. The isoform-specific regulation of apoptosis by protein kinase C. CMLS, Cell. Mol. Life Sci. 60, 1061-1070 (2003).

41. Myers, K., Gagou, M. E., Zuazua-Villar, P., Rodriguez, R. \& Meuth, M. ATR and Chk1 Suppress a Caspase-3-Dependent Apoptotic Response Following DNA Replication Stress. PLoS Genet 5, e1000324 (2009)

42. Shackelford, D. B. \& Shaw, R. J. The LKB1-AMPK pathway: metabolism and growth control in tumour suppression. Nat Rev Cancer 9, 563-575 (2009).

43. Wang, Z. et al. Cyclin-dependent kinase 1-mediated phosphorylation of YES links mitotic arrest and apoptosis during antitubulin chemotherapy. Cellular Signalling 52, 137-146 (2018).

44. Gu, J. J., Wang, Z., Reeves, R. \& Magnuson, N. S. PIM1 phosphorylates and negatively regulates ASK1-mediated apoptosis. Oncogene 28, 4261-4271 (2009). 
45. Luan, W. et al. Long non-coding RNA MALAT1 acts as a competing endogenous RNA to promote malignant melanoma growth and metastasis by sponging miR-22. Oncotarget 7, 63901-63912 (2016).

46. Wang, W.-T., Han, C., Sun, Y.-M., Chen, T.-Q. \& Chen, Y.-Q. Noncoding RNAs in cancer therapy resistance and targeted drug development. J Hematol Oncol 12, 55 (2019).

47. Wright, C. J. M. \& McCormack, P. L. Trametinib: First Global Approval. Drugs 73, 1245-1254 (2013).

48. Vasan, N., Baselga, J. \& Hyman, D. M. A view on drug resistance in cancer. Nature 575, 299-309 (2019).

49. Sawyers, C. L. Perspective: Combined forces. Nature 498, S7-S7 (2013).

50. Kling, J. Bundling next-generation cancer therapies for synergy. Nat Biotechnol 24, 871-872 (2006).

51. Kasuya, T. et al. Ribonuclease H1-dependent hepatotoxicity caused by locked nucleic acidmodified gapmer antisense oligonucleotides. Sci Rep 6, 30377 (2016).

52. Zheng, Z.-M. Development of Resistance to RNAi in Mammalian Cells. Annals of the New York Academy of Sciences 1058, 105-118 (2005).

53. Hodis, E. et al. A landscape of driver mutations in melanoma. Cell 150, 251-263 (2012).

54. Wu, H. et al. Comprehensive bioinformatics analysis of critical IncRNAs, mRNAs and miRNAs in non-alcoholic fatty liver disease. Mol Med Report (2019) doi:10.3892/mmr.2019.9931.

55. Gene Therapy of Pancreatic Ductal Adenocarcinoma (TherGAP). https://clinicaltrials.gov/ct2/show/NCT01274455.

56. IFx-Hu2.0 for the Treatment of Patients With Skin Cancer.

https://clinicaltrials.gov/ct2/show/NCT04925713.

57. IFx-Hu2.0 Expanded Access Program. https://clinicaltrials.gov/ct2/show/NCT04853602. 
58. Immunotherapy With IFx-Hu2.0 Vaccine for Advanced Non-melanoma Skin Cancers.

https://clinicaltrials.gov/ct2/show/NCT04160065.

59. Leucci, E. et al. Melanoma addiction to the long non-coding RNA SAMMSON. Nature 531, 518522 (2016).

60. Zehntner, S. P. et al. X-linked Inhibitor of Apoptosis Regulates T Cell Effector Function. J Immunol 179, 7553-7560 (2007).

61. Frankish, A. et al. GENCODE 2021. Nucleic Acids Research 49, D916-D923 (2021).

62. O'Leary, N. A. et al. Reference sequence (RefSeq) database at NCBI: current status, taxonomic expansion, and functional annotation. Nucleic Acids Res 44, D733-D745 (2016).

63. Kittler, R. et al. Genome-wide resources of endoribonuclease-prepared short interfering RNAs for specific loss-of-function studies. Nat Methods 4, 337-344 (2007).

64. Lonsdale, J. et al. The Genotype-Tissue Expression (GTEx) project. Nat Genet 45, 580-585 (2013).

65. Kinome Activity Mapping, Coppé, J.-P., Mori, M. \& Pan, B. High-Throughput Kinase Activity Mapping (HT-KAM) system: biochemical assay. Protocol Exchange (2019) doi:10.1038/protex.2019.029.

66. Coppé, J.-P., Yau, C. \& Wolf, D. M. High-Throughput Kinase Activity Mapping (HT-KAM) system: analysis of phospho-catalytic profiles. Protocol Exchange (2019) doi:10.1038/protex.2019.030.

67. Huang, D. W., Sherman, B. T. \& Lempicki, R. A. Systematic and integrative analysis of large gene lists using DAVID bioinformatics resources. Nat Protoc 4, 44-57 (2009).

68. Huang, D. W., Sherman, B. T. \& Lempicki, R. A. Bioinformatics enrichment tools: paths toward the comprehensive functional analysis of large gene lists. Nucleic Acids Research 37, 1-13 (2009). 


\section{Figure Legends}

\section{Figure 1. The IncRNA AC004540.4 (TRASH) is responsive to MAPK-activation and essential for}

melanoma cell-survival a) Schematic draft of pipeline steps to identify MAPK responsive IncRNAs that are essential for melanoma cell-survival. NRAS mutant primary human melanocytic $\left(\mathrm{PHM}^{\mathrm{Q} 61}\right)$ and NRASmutant melanoma cell-lines (D04, MM415) were compared to wild type primary human melanocytic cell-lines (PHM $\left.{ }^{\mathrm{E}}, \mathrm{PHM}\right)$. Differential expressed (DE) genes were filtered for up-regulated IncRNAs, high occurrence (>90\%) in TCGA patient samples and significant contribution to melanoma cell-survival b) Transcriptome intersect of DE genes of the three comparisons $\mathrm{PHM}^{\mathrm{Q} 61} / \mathrm{PHM}^{\mathrm{E}}, \mathrm{D} 04 / \mathrm{PHM}$ and MM415/PHM displayed in a Venn diagram. Genes were considered as DE, when absolute value of the expression change was greater than 1 log2-fold. 197 DE genes were filtered, of which 81 were upregulated IncRNA genes. c) Expression change in all three comparisons of the 197 DE genes from the filter step in panel b) displayed in a heatmap. Genes were classified in the subgroups of TUCP (transcript of unknown-coding potential), RNA genes and Protein-coding genes. 81 of the 197 DE genes were upregulated IncRNAs d) Filter for potential clinically relevant targets. A scatter chart diagram shows that 24 of the 81 up-regulated DE IncRNAs from panel c) were expressed in >90\% (grey bar) of NRAS-mutant melanoma patients in TCGA-SKCM dataset. LncRNA genes were ranked from 1 (lowest) to 81 (highest) average FPKM-values. The IncRNA TRASH is highlighted in red. e) Filter for relevance in melanoma cellsurvival. Expression of the 24 IncRNAs that were filtered out in panel d) was silenced using RNAi libraries, leading to significant cell-growth inhibition effects for 6 targets. Bar graphs display esiRNA (endoribonuclease prepared siRNA, top) and siRNA (bottom) mediated silencing of the top candidate TRASH. TRASH-targeting RNAi significantly reduced cell-growth in the melanoma cell-lines D04 (esiRNA: $p<0.001$, siRNA: $p=0.027$ ), MM415 (esiRNA: $p<0.001$, siRNA: $p=0.002$ ), WM3629 (esiRNA: $p=0.001$, siRNA: $p=0.001$ ) and Sk-Mel-28 (esiRNA: $p=0.009$, siRNA: $p=0.007$ ) but not in primary human melanocytic cell-lines (esiRNA: $p=0.426$, siRNA: $p=0.171$ ). Data were normalized to treatment with non- 
targeting pooled control siRNA (Control), cells were incubated in media with 50nM oligonucleotide concentration for 72 hours ( $n=3)$. ATP quantification was used as marker for metabolically active cells. Error-bars represent standard deviation, significance is shown as p-values calculated by Students t-test. $*=p<0.05, * *=p<0.01, * * *=p<0.001$.

Figure 2. AC004540.4 (TRASH) is nuclear enriched, inefficiently spliced and interacts with hnRNPA2/B1. a) Subcellular enrichment analysis of TRASH. Nuclear/cytoplasmic ratio of RNA expression of the IncRNAs TRASH (nuclear), H19 (cytoplasmic) and MALAT1 (nuclear) normalized to the IncRNA Nuclear Enriched Antisense Transcript 1 (NEAT1) in the D04 melanoma cell-line $(n=3)$. Data are shown as log2 of fold enrichment. The IncRNAs MALAT1 and H19 served as control. b) TRASH exists in inefficiently spliced forms. qRT-PCR analysis showing relative fold enrichment of 4 different transcript regions of TRASH using different primer pairs that target Exon 1 (1), Intron 1 (2), the Intron1/Exon2 transition region (3) and Exon 2 (4) of the Isoform ENST00000451264.1 in D04 cells. Fold enrichment was calculated using the $2^{-\Delta \Delta C t}$ method and normalized to primer pair $4(n=3)$. c) TRASH and hnRNPA2/B1 expressions are upregulated in melanoma. Gene expression of TRASH and hnRNPA2/B1 is significantly $(* * *=p<0.001)$ up-regulated in TCGA-SKCM melanoma samples $(n=469)$ when compared to GTEx patient samples of non-malignant skin biopsies $(n=394)$. Significance is shown as $p$-values calculated by Students t-test. d) TRASH-ASO treatment significantly inhibits TRASH, but not hnRNPA2/B1 gene expression. QRTPCR analysis showing relative fold enrichment of TRASH and hnRNPA2/B1 in the D04 cell-line after 1 day of TRASH-ASO or Control-ASO treatment. Treatment period was 1 day in $50 \mathrm{nM}$ ASO concentration $(n=3)$. Relative gene expression was normalized to Control-ASO treatment. Fold-change cut off for significant expression-inhibition was considered as 0.5 (blue bar). e) TRASH-ASO treatment reduces hnRNPA2/B1 protein levels. Immunoblotting showing strongly decreased hnRNPA2/B1 protein levels upon 1- and 2days after TRASH-ASO treatment versus Control-ASO treatment $(100 \mathrm{nM})$ in lysate of D04 cells. $\beta$-actin 
served as loading control. f) RNA-binding Protein Immunoprecipitation unveils that TRASH binds to hnRNPA2/B1. Top: Immunoblotting showing enrichment of hnRNPA2/B1 protein in hnRNPA2/B1 pulldown samples compared to normal rabbit IgG pulldown control. $\beta$-actin served as loading control. Bottom: qRT-PCR analysis showing >65-fold enrichment relative fold enrichment of AC004540.4 (TRASH) in pulldown samples of hnRNPA2/B1 compared to normal Rabbit IgG control pull down $(n=3)$. Error bars represent standard deviation.

Figure 3. TRASH-ASO treatment is anti-proliferative, pro-apoptotic and globally affects gene expression in melanoma. a) TRASH-ASO treatment inhibits cell-growth in melanoma. TRASH-ASO treatment leads to significant reduction of cell growth in the D04 $(p<0.001), M M 415(p<0.001)$, WM1366 ( $p=0.009)$, VMM39 ( $p=0.002)$, Sk-Mel-2 ( $p<0.001)$, WM3629 $(p<0.001)$, Sk-Mel-28 $(p=0.003)$, WM3211 $(p=0.006)$ melanoma cell-lines, the Hs852.T $(p<0.001)$ and AV5 $(p<0.001)$ primary melanoma cell-lines, but not in the pooled primary human melanocytic cell-lines PHM $(p=0.356)$. Cell-growth was normalized to treatment with Control-ASOs. Cells were incubated in media with 50nM ASO concentration for five days $(n=3)$. b) TRASH-ASO treatment inhibits colony formation in melanoma. Left: TRASH-ASO treatment leads to significantly reduced colony formation in the D04 ( $p=0.002)$, MM415 $(p=0.004)$ and Sk-Mel-28 ( $p=0.002)$ melanoma cell-line, compared to treatment with Control-ASOs. Cells were incubated in media with 50nM ASO concentration for seven days ( $n=3)$. Right: Representative image of colony formation in $6 \mathrm{~cm}$ dishes after TRASH-ASO and Control-ASO treatment in the D04 melanoma cell-line. c) HnRNPA2/B1-ASO treatment significantly $(p=0.001)$ inhibits cell-growth in the D04 melanoma cell-lines, but to a lesser extent than TRASH-ASO treatment when compared to panel a). Cell-growth was normalized to treatment with Control-ASOs, cells were incubated in media with 50nM ASO concentration for five days $(n=3)$. d) TRASH-ASO treatment induces apoptosis to a stronger extent than hnRNPA2/B1-ASO treatment. Activity levels of the apoptosis markers Caspase- $3 \&-7$ are 
significantly increased upon TRASH- $(p=0.001)$, and hnRNPA2/B1-ASO $(p<0.001)$ treatment $(50 n M)$ in the D04 cell-line. Caspase activity was normalized to treatment with Control-ASOs (50nM). Incubation time was one day $(n=4)$. e) TRASH-ASO treatment has a global effect on gene expression. Scatter plot diagram showing differential gene expression after TRASH-ASO treatment compared to Control-ASO treatment. (cut-off for significance was adjusted p-value < 0.05). Data was obtained from RNA-Seq of D04 melanoma cells, treatment period was three days. In a+c ATP quantitation was used as marker for metabolically active cells. Significance is shown as $p$-values calculated by Students t-test. ${ }^{*}=p<0.05$, $* *=p<0.01, * * *=p<0.001$. Error bars represent standard deviation.

Figure 4. Mapping the phospho-catalytic signatures of TRASH-dependent cells identifies inhibition of anti-apoptotic kinases upon TRASH-ASO treatment. a) Peptide-associated phosphorylation profiles of melanoma cell-lines treated with Control-ASOs or TRASH-ASOs for one day (50nM). Unsupervised clustering was applied (uncentered correlation and average linkage for both peptides/horizontal and samples/vertical). The profile of each sample is the average of two independent assay repeats. b) Kinase activity signatures of melanoma cell-lines treated with Control-ASOs, or TRASH-ASOs for one day (50nM). Kinases signatures are derived from results shown in panel a). Kinases for which $\geq 3$ biological peptides are available, are shown. Unsupervised clustering was applied as in panel a). c) Kinase activity profiles of a subset of kinases known to promote cell-survival by preventing apoptosis. Kinase activities are normalized to Control-ASO treatment per cell-line. The effect of TRASH-ASOs on these kinases is compared side-by-side to MALAT1-ASO treatment. d) MALAT1-ASO treatment inhibits cell-growth and induces apoptosis. Left: Cell-growth is significantly $(p<0.001)$ decreased upon MALAT1-ASO treatment $(50 \mathrm{nM})$ in the D04 cell-line. Cell-growth is relative to Control-ASO treatment (50nM). Incubation time was five days ( $n=3)$. ATP quantitation was used as marker for metabolically active cells. Right: Activity levels of the apoptosis markers Caspase-3 \& -7 are significantly $(p=0.003)$ upregulated upon MALAT1- 
ASO treatment (50nM) in the D04 cell-line. Caspase activity was normalized to treatment with ControlASOs (50nM). Incubation time was one day $(n=4)$. and significance is shown as $p$-values calculated by Students t-test. ${ }^{*}=p<0.05,{ }^{* *}=p<0.01,{ }^{* * *}=p<0.001$. Error bars represent standard deviation. e) The specificity of the effects of TRASH-ASO treatment on the kinase activity signatures of melanoma cells is assessed in comparison to MALAT1-ASO treatment using Pearson correlation. f) Schematic summarizing the molecular impact of TRASH-ASO treatment. Expression of the IncRNA TRASH is an essential dependency that promotes the survival of melanoma cells, and that can be effectively targeted with ASOs.

\section{Figure 5. TRASH-ASO treatment has potential clinical value and reduces tumor growth in-vivo. a)}

TRASH-ASO treatment inhibits cell-growth in the MEKi-resistant melanoma. TRASH-ASO treatment leads to significant reduction of cell growth in the trametinib treatment resistant D04RM $(p<0.001)$, MM415RM ( $p<0.001)$, WM3629RM $(p<0.001)$ and Sk-Mel-2RM ( $p=0.002)$ cell-lines. Cell-growth was normalized to treatment with Control-ASOs, cells were incubated in media with 50nM ASO concentration for five days $(n=3)$. Cell-growth reduction is comparable to their non-resistant treatmentnaïve cell-line counterparts shown in panel 3a) b) TRASH-ASO and MEKi combinations confer synergistic effects. Dual treatment with TRASH-ASO (25 and 50nM) and the MEKi (trametinib, $0.2-100 \mathrm{nM}$ ) leads to reduced cell-growth and synergistic effects (Combination Index $>0$ ) in the D04 and AV5 melanoma cellline. Treatment period was three days $(n=2)$. c) In contrast to MEKi, TRASH-ASO treatment does not lead to drug resistance. D04 Cells that survived initial TRASH-ASO (50nM) or MEKi (15nM) treatment subsequently recovered in drug free media. Repetition of the preceding drug treatment in the surviving cell-subpopulation (same conditions) led to significantly increased ( $p=0.004)$ cell-growth reduction for TRASH-ASO treatment and significantly decreased $(p<0.001)$ cell-growth reduction for MEKi treatment. Cell-growth is relative to incubation with Control-ASOs (TRASH) or drug free media (MEKi). Drug- 
incubation time was five days $(n=3)$. ATP quantitation was used as marker for metabolically active cells.

d) Systemic TRASH-ASO treatment inhibits tumor growth in vivo and does not cause toxicity. Top:

Significant tumor growth reduction upon TRASH-ASO treatment was observed in mouse models carrying xenografts of the D04 melanoma cell-line, the AV5 primary melanoma cell-line and the TM01341 patient-derived tumor (PDX). Bottom: No significant difference in weight change in comparison of TRASH and Control groups could be observed for any of the three tumor models. Mice received systemic TRASH-ASO or Control-ASO treatment (2x/week). Growth and weight curves are displayed as polynomial trendlines (Order: 2) e) TRASO-ASO treatment inhibits TRASH expression in vivo. qRT-PCR analysis showing relative fold enrichment of TRASH in RNA extracts of D04 tumors. TRASH expression was significantly reduced after 21 days of systemic ASO treatment. $(n=2)$. Relative gene expression was normalized to RNA extracts of Control-ASO treated D04 tumors. Fold-change cut off for significant expression-inhibition was considered as 0.5 (blue bar). e) TRASH-ASO treatment induces apoptosis and does not cause hepatotoxicity in vivo. Left: Immunohistochemical staining for the expression of the apoptosis marker Cleaved-Caspase-3 in D04 tumors after 21 days of TRASH-ASO (bottom) and ControlASO treatment (top). Right: Hematoxylin-eosin staining of mouse liver tissue after 21 days of TRASH-ASO (bottom) and Control-ASO treatment (top). Significance in a), c) and d) is shown as p-values calculated by Students t-test. ${ }^{*}=p<0.05,{ }^{* *}=p<0.01,{ }^{* * *}=p<0.001$. Error bars represent standard deviation.

\section{Supplementary Figure 1. Generating melanocytic cell-lines with NRAS ${ }^{\mathrm{Q} 61}$ mutation and detailed}

bioinformatics pipeline workflow. a) From left to right: Visual representation of the pENTR/D-TOPO plasmid that was used to create an NRAS mutant melanocytic cell-line $\left(\mathrm{PHM}^{\mathrm{Q} 61}\right)$, showing the rrnB_T2 and rrnB_T1 terminator sequences, the attL1 and attL2 recombination sites, the green fluorescent protein sequence (GFP), the kanamycin resistance gene pUC origin of replication sequence and the NRAS gene harboring a Q61 mutation in Codon 61. Sanger-Sequencing verified successful insert of an NRAS ${ }^{061}$ 
mutation in $\mathrm{PHM}^{\mathrm{Q} 61}$ but not in melanocytic cell-lines that were transfected with an empty vector $\left(\mathrm{PHM}^{\mathrm{E}}\right)$. 20x Microscopic imaging of $\mathrm{PHM}^{\mathrm{Q} 61}$ and $\mathrm{PHM}^{\mathrm{E}}$ with co-expressed green fluorescent protein that was used as transduction efficacy reporter. Immunoblotting showing induced MAPK signaling in form of upregulation of NRAS an the NRAS downstream signalling effectors $p$-AKT and $p$-ERK in PHM ${ }^{\mathrm{Q} 61}$ compared to $\mathrm{PHM}^{\mathrm{E}}$ cells. GAPDH served as loading control. $\mathrm{PHM}^{\mathrm{E}}$ and $\mathrm{PHM}^{\mathrm{Q} 61}$ showed no significant differences in cell proliferation. ATP quantitation was used as marker for metabolically active cells and measured five days after seeding equal number of cells ( $n=3)$. $p$-value was calculated using Students t-test. b) Flowchart showing individual bioinformatic pipeline steps for comparing and analysing RNA-Seq results to identify IncRNA genes that respond with upregulation to activation of MAPK signalling. c) Flowchart showing final steps to identify TRASH and five other relevant IncRNAs that are responsive to MAPK upregulation and essential for melanoma cell survival. The steps included the integration of RNA-Seq data of 86 NRAS mutant patient-derived tumor samples from The Cancer Genome Atlas (TCGA) to filter for clinically relevant targets and RNAi screening to filter for their relevant role for cell survival.

Supplementary Figure 2. AC004540.4 (TRASH) annotation in genomic databases and RNA-expression of AC004540.4 (TRASH) and hnRNPA2/B1 in patients. a) As annotated in LNCipedia, species conservation and the coding probability scores for TRASH Ensembl Transcript IDs ENST00000451264 and ENST00000451368. TRASH is not conserved in other species and identified as non-coding transcript by five different informatic tools. b-e) TRASH and hnRNPA2/B1 expression correlates in melanoma but not in non-malignant skin. Spearman correlation of RNA-expression of TRASH and hnRNPA2/B1 in melanoma $(\rho=0.41, b+c)$ and non-malignant skin tissue $(\rho=0.24, d+e)$ is displayed as red line. Black dots represent Spearman correlation of TRASH, b+d or hnRNPA2/B1 (c+e) in comparison to 10 sets of 200 random genes. Melanoma data are derived from the TCGA-SKCM dataset $(n=469)$, and non-malignant 
skin tissue data are derived from the GTEx dataset $(n=394)$. Significance is shown as $p$-values calculated by Students t-test. ${ }^{*}=p<0.05,{ }^{* *}=p<0.01,{ }^{* *}=p<0.001$. Error bars represent standard deviation. 


\section{melanoma}

\section{Main Figures 1-5}

\&

Extended Data Figures 1-2

Figure legends are listed in the manuscript document 
Main Figures 
a

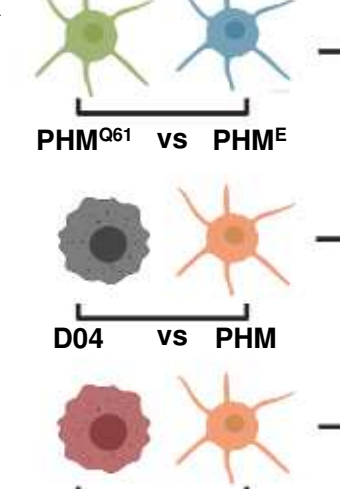

MM415 vs PHM
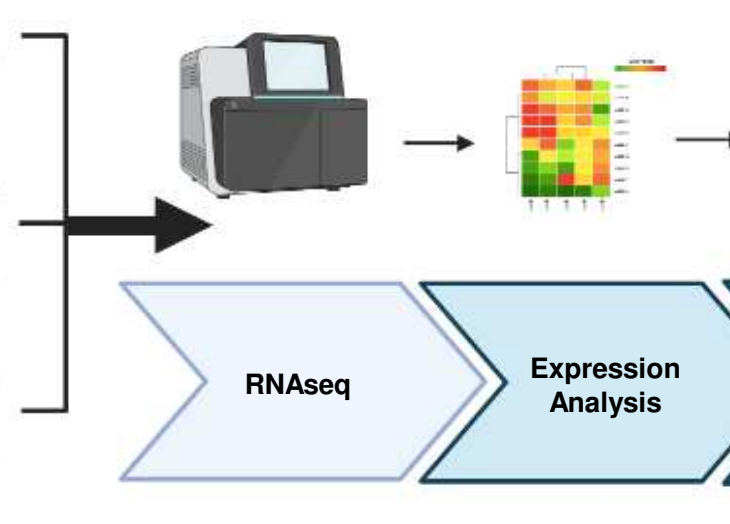

Expression Analysis

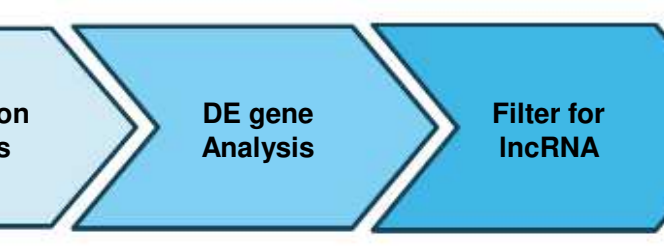

$\rightarrow$ TCGA\&B $\rightarrow=-\ldots$

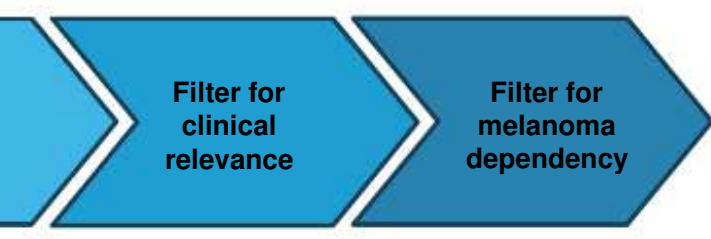

c
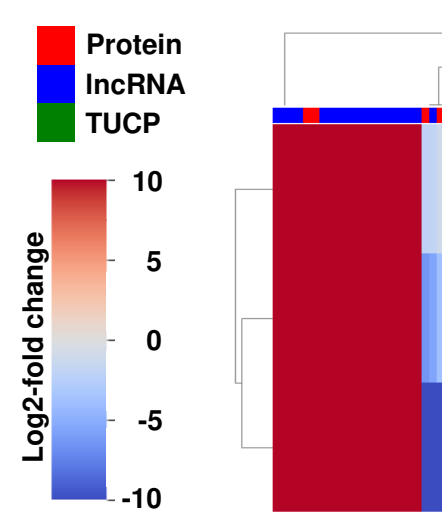

d

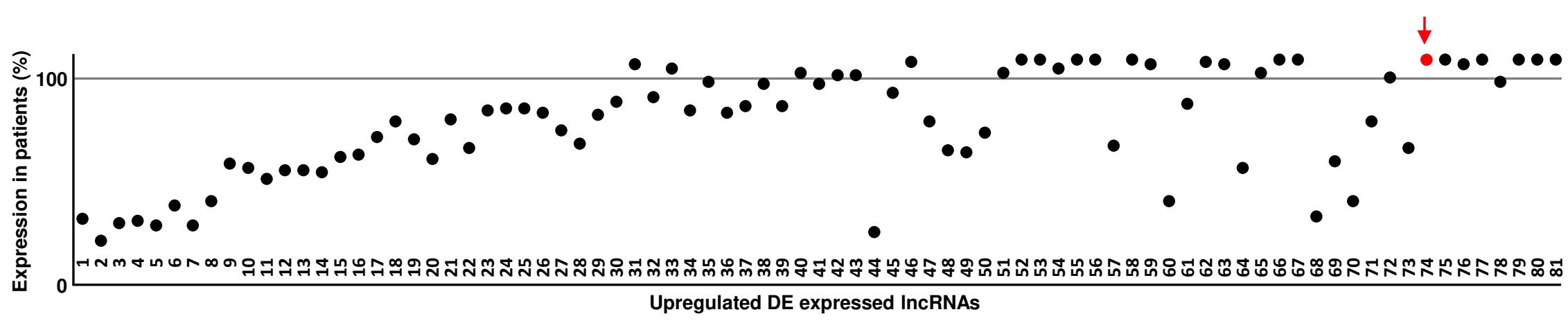

b
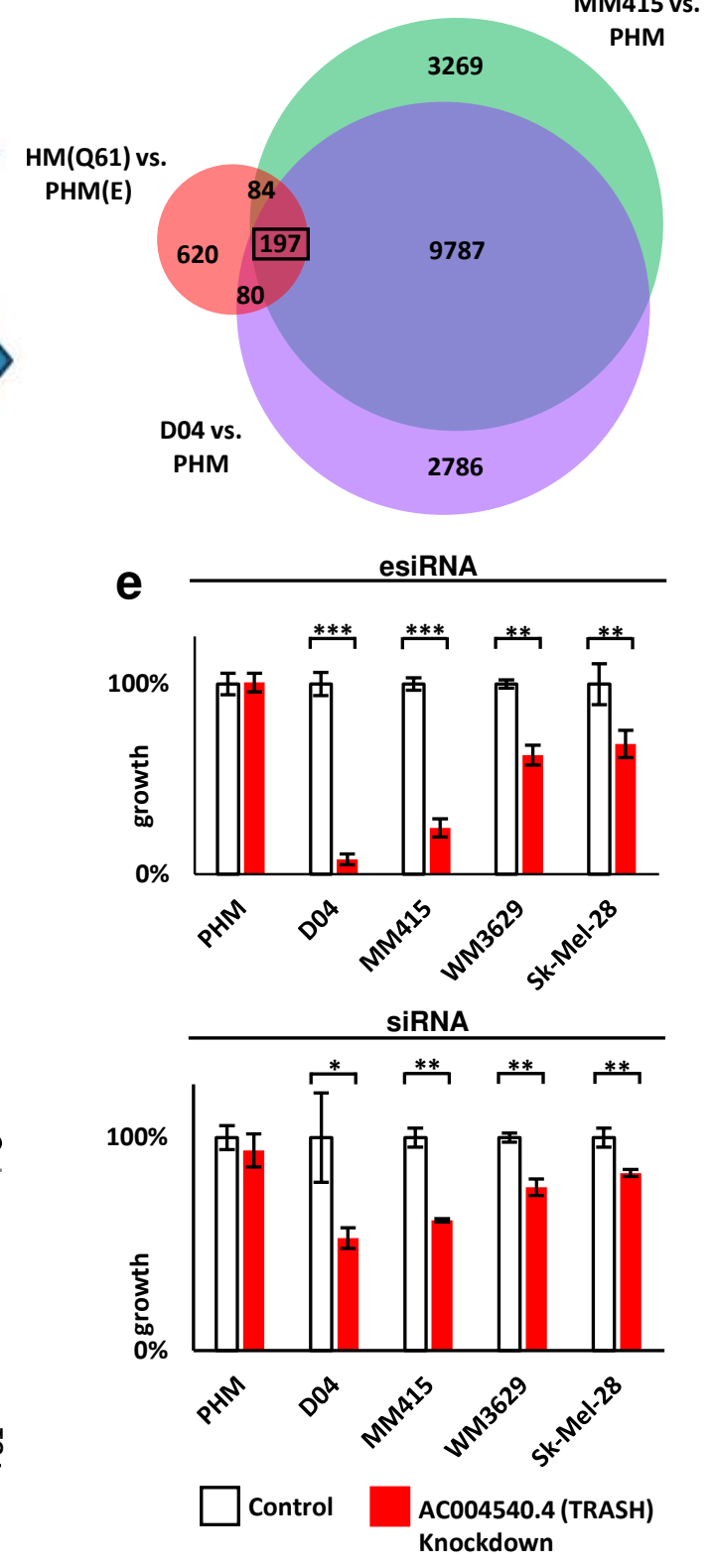

Figure 1 


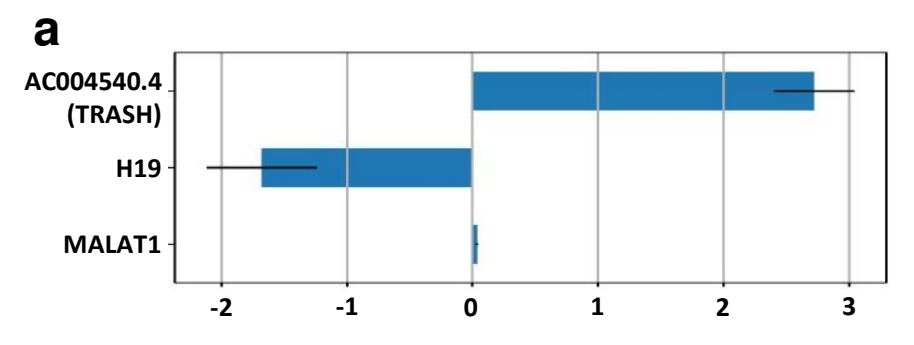

Log2-fold expression change of nuclear IncRNA expression

d

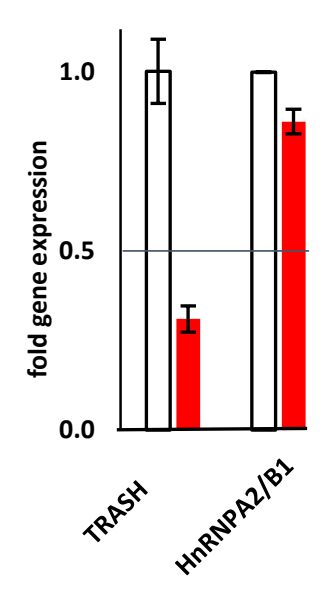

$\square$ Control-Aso

TRASH-ASO rmalized to NEAT1 b

AC004540.4 (TRASH) Transcript ENST00000451264.1

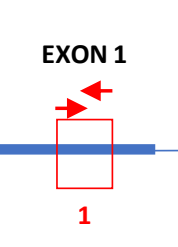

elative $\mathrm{AC} 004540.4$ (TRASH) fold nrichment of primer

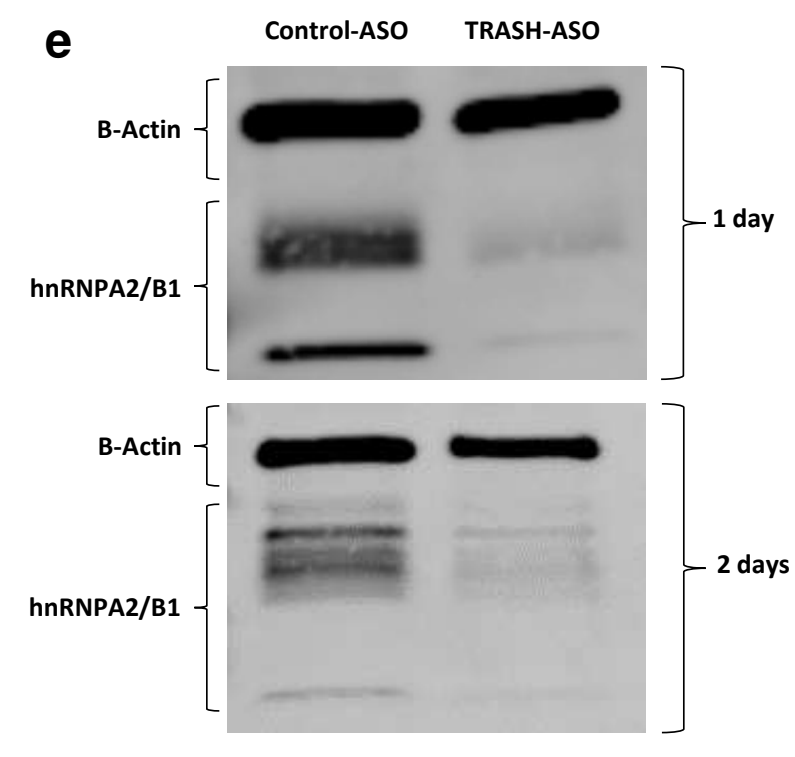

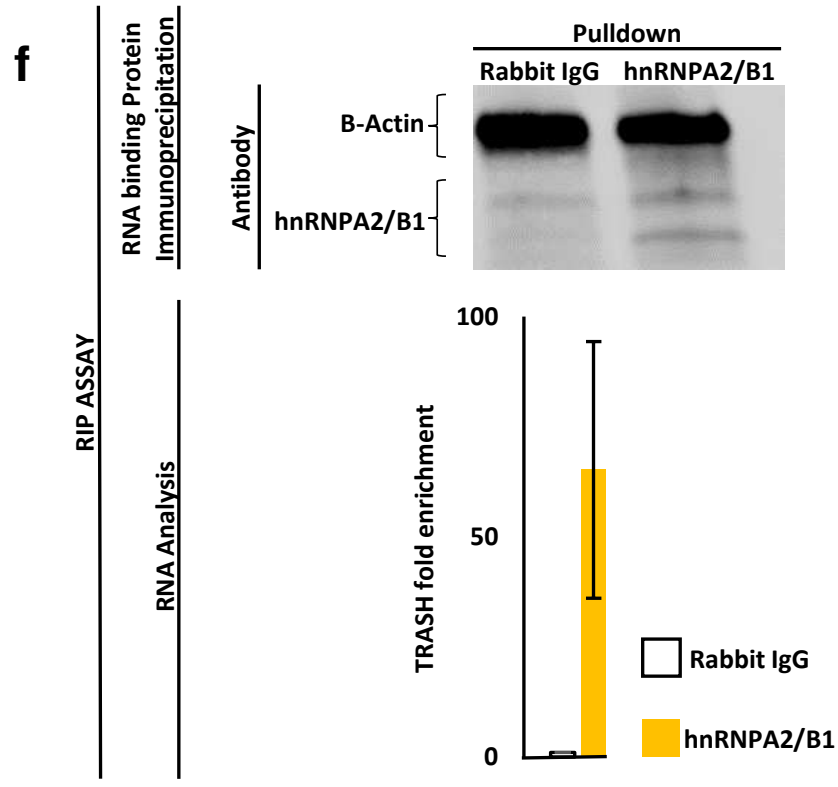

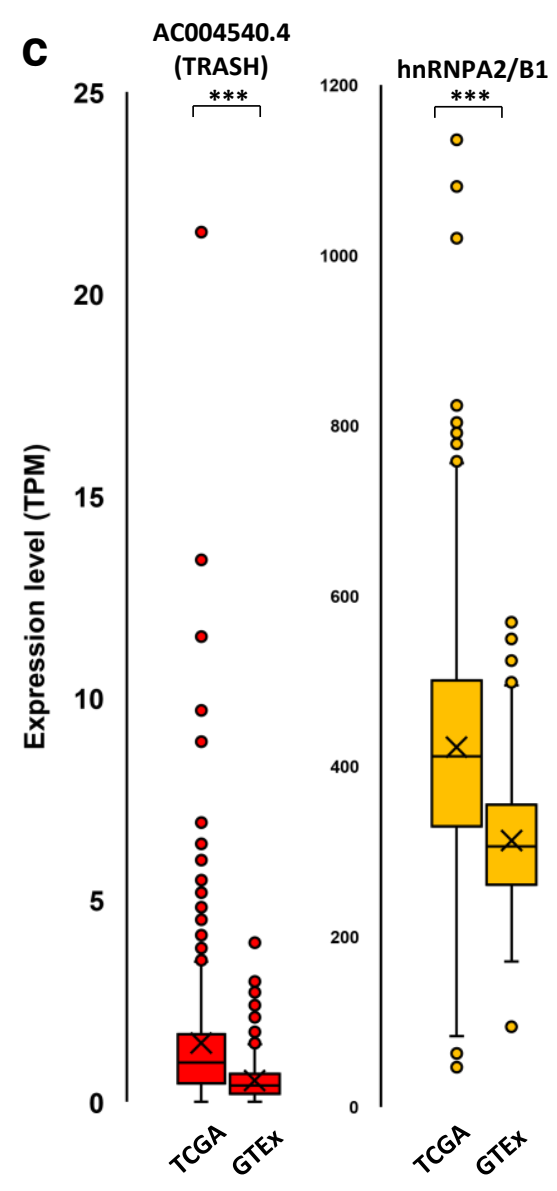


a
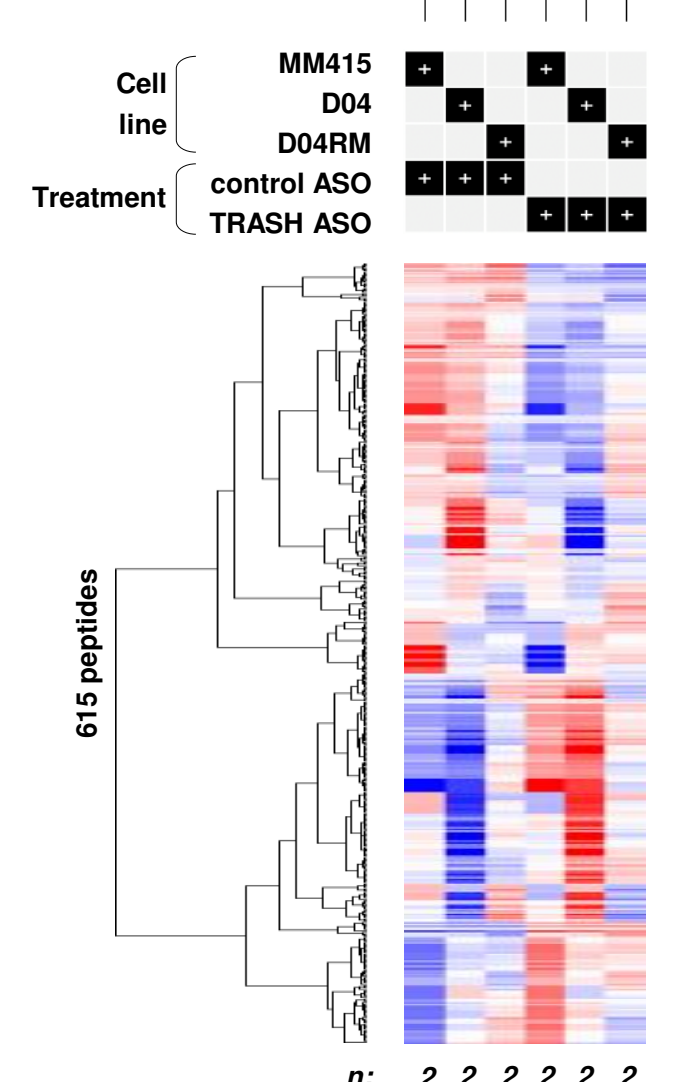

n: 22222222

Peptide-associated phosphorylation activity scale (ATP consumption in $\mathrm{nM}$ )

$$
-20 \text { + } 20
$$

mean-centered per cell line on the average activity measured in
control ASO and TRASH ASO samples b
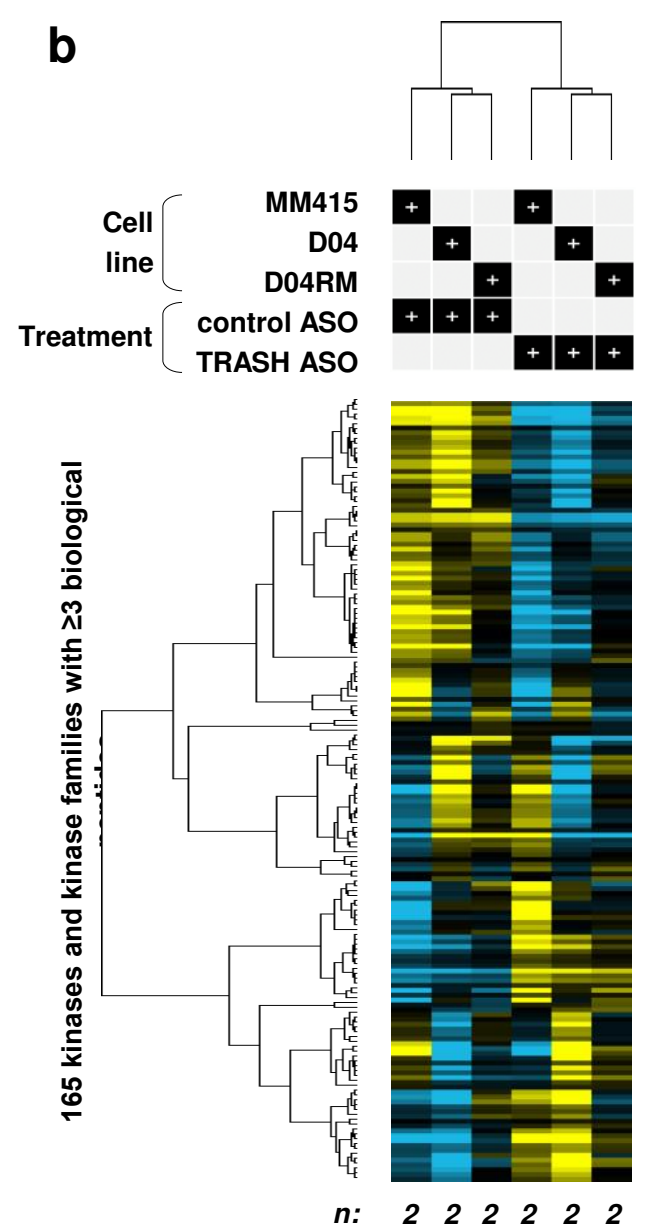

n: $\quad 2 \quad 2 \quad 2 \quad 2 \quad 2 \quad 2$

Kinase phospho-catalytic activity scale (nM ATP)

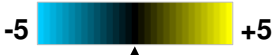

mean-centered per cell line on the average activity measured in control ASO and TRASH ASO samples
C

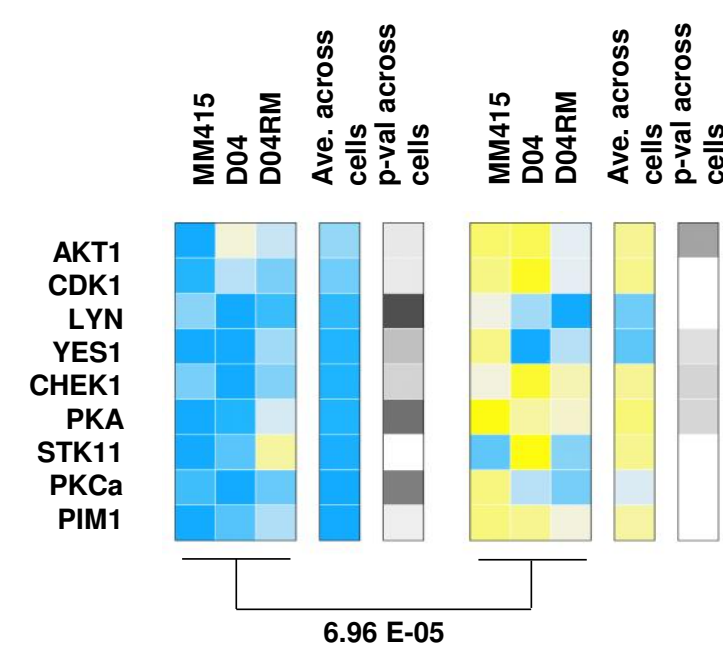

$\overbrace{\text { mean-centered on }}^{\uparrow}+5$

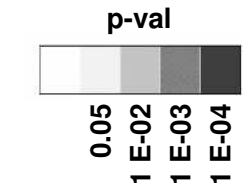

the activity measured

in control ASO

d

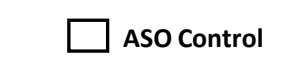

ASO Malat1
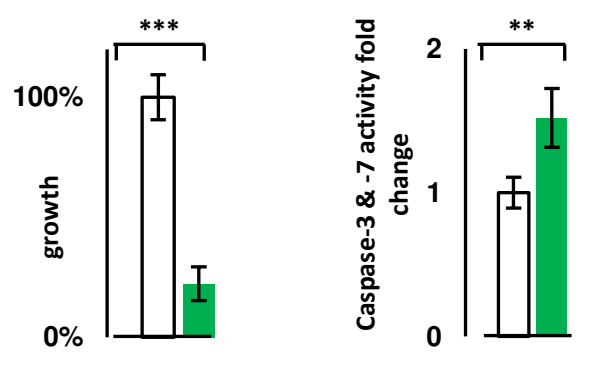

e

kinase signatures

vs. MALAT1 ASO

vs. MALAT1 ASO

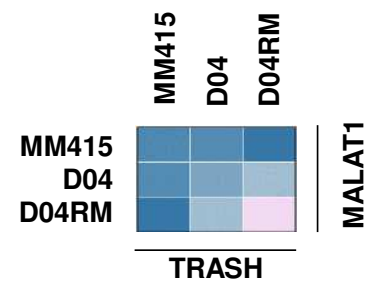

Pearson correlation (r)

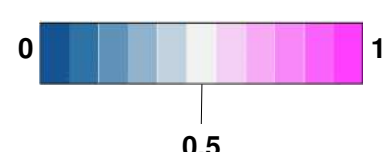

0.5

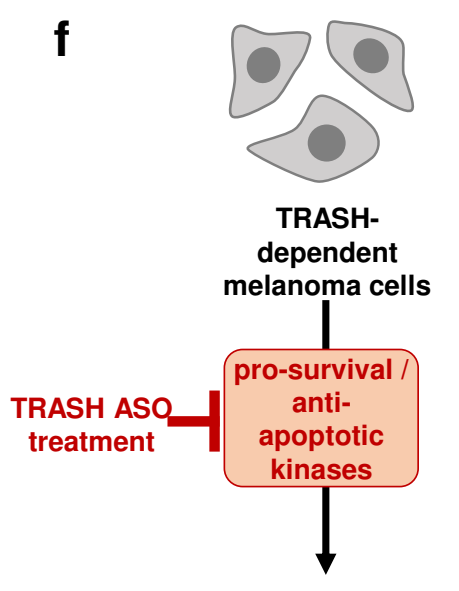

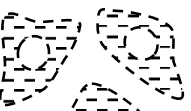



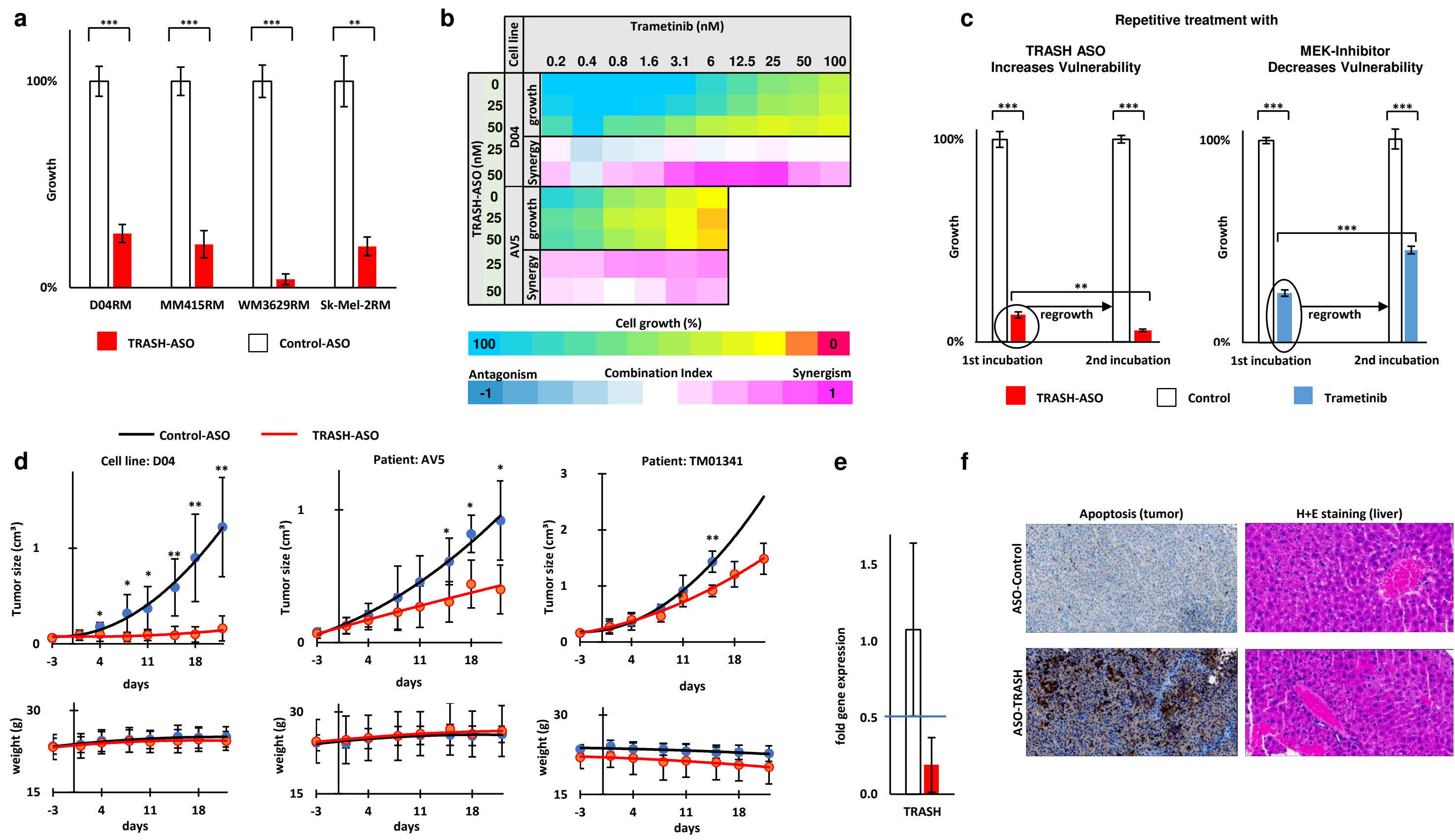
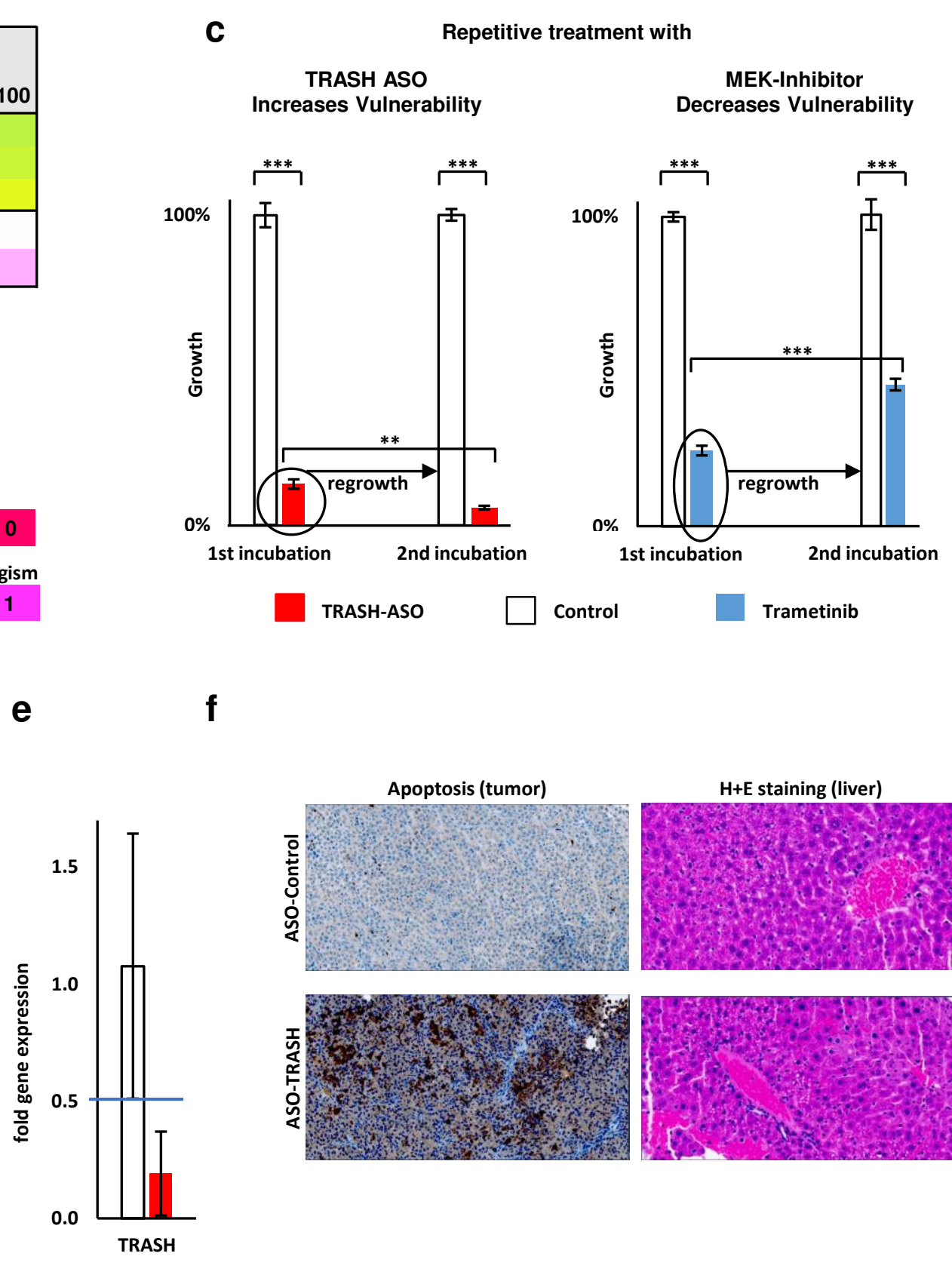

e

f 


\section{Extended Data Figures}




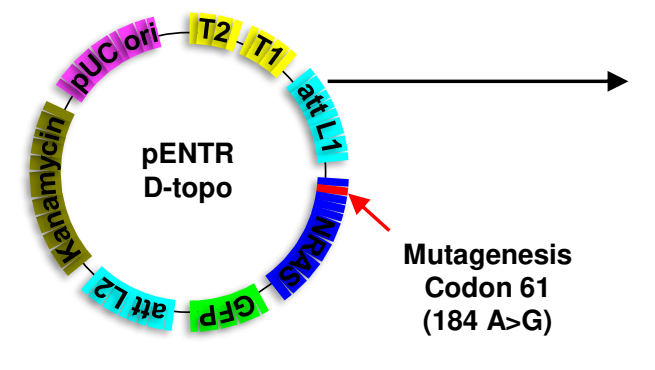

PHME

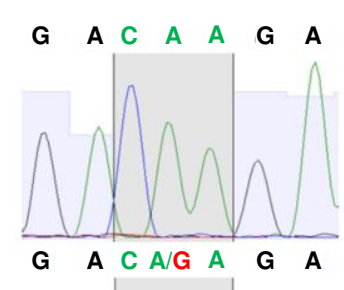

PHM ${ }^{061}$ (184 A>G)

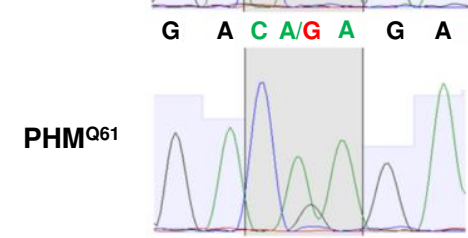

PHME

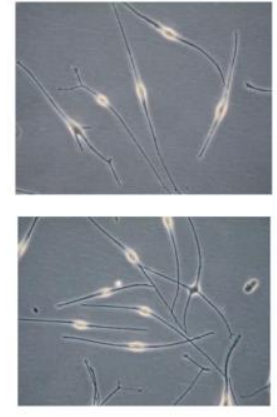

20x

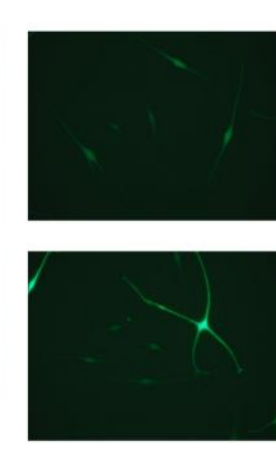

20x GFP
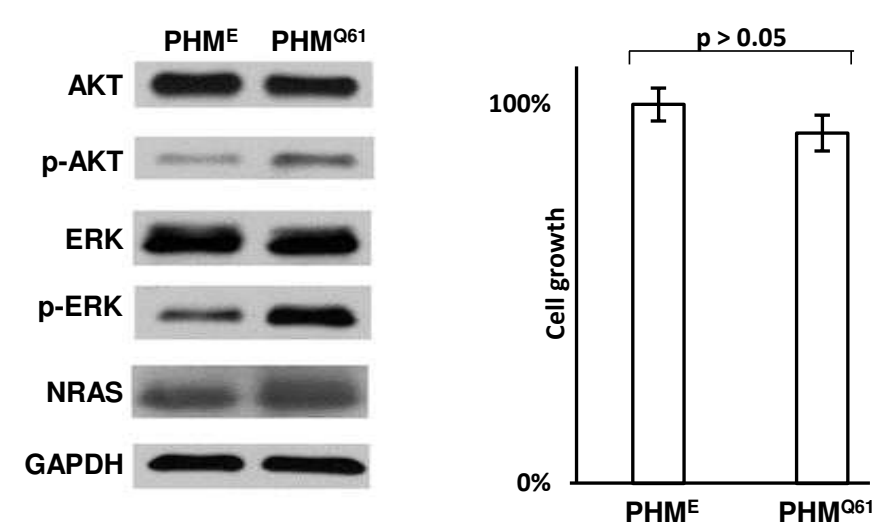

Bioinformatic pipelin

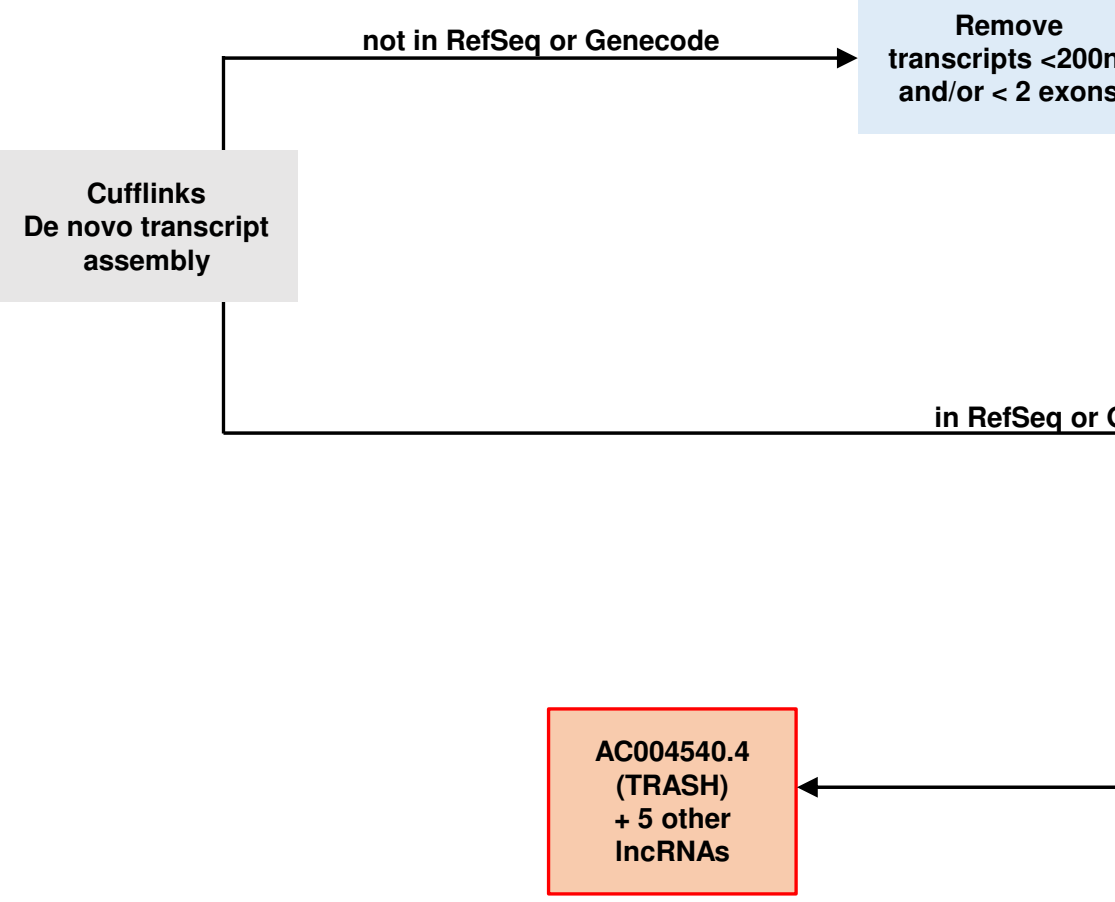

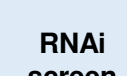

screen
24 DE

RNA gene IDs
Remove gene IDs expressed in $<90 \%$ of TCGA samples

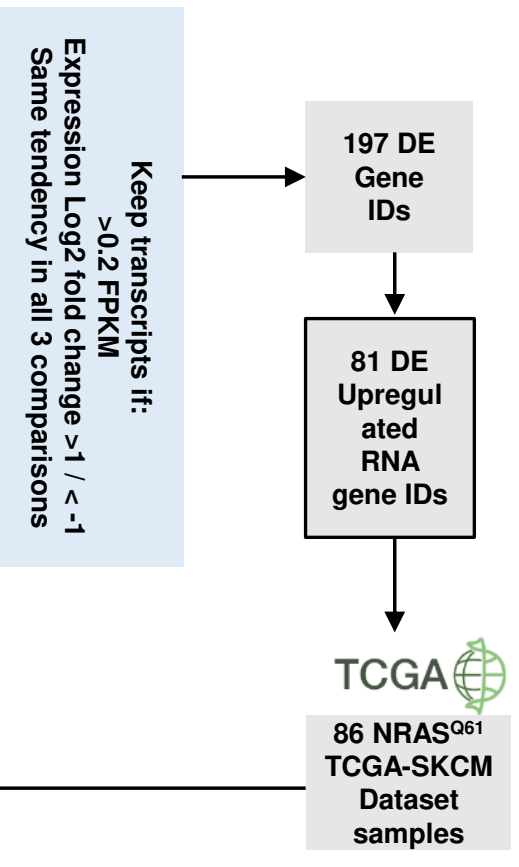


a

\begin{tabular}{|c|c|c|c|}
\hline \multicolumn{2}{|c|}{ Protein coding potential metrics } & \multirow{2}{*}{$\begin{array}{c}\text { Isoform: } \\
\text { ENST00000451264 } \\
0 \\
\end{array}$} & \multirow{2}{*}{$\begin{array}{c}\text { Isoform: } \\
\text { ENST00000451368 } \\
0 \\
\end{array}$} \\
\hline PRIDE & Raw result & & \\
\hline reprocessing 2.0 & Interpretation & non-coding & non-coding \\
\hline \multirow{2}{*}{$\begin{array}{l}\text { Lee translation } \\
\text { initiation sites }\end{array}$} & Raw result & 0 & 0 \\
\hline & Interpretation & non-coding & non-coding \\
\hline \multirow{2}{*}{ PhyloCSF score } & Raw result & -14.2375 & -14.2375 \\
\hline & Interpretation & non-coding & non-coding \\
\hline \multirow{2}{*}{$\begin{array}{l}\text { CPAT coding } \\
\text { probability }\end{array}$} & Raw result & $0.17 \%$ & $3.41 \%$ \\
\hline & Interpretation & non-coding & non-coding \\
\hline \multirow{2}{*}{$\begin{array}{c}\text { Bazzini small } \\
\text { ORFs }\end{array}$} & Raw result & 0 & 0 \\
\hline & Interpretation & non-coding & non-coding \\
\hline \multicolumn{4}{|c|}{ Locus conservation } \\
\hline \multirow{2}{*}{\multicolumn{2}{|c|}{$\frac{\text { Fruitfly }}{\text { Zebrafish }}$}} & No & No \\
\hline & & No & No \\
\hline \multicolumn{2}{|c|}{ Mouse } & No & No \\
\hline \multicolumn{2}{|c|}{ Chimpanzee } & No & No \\
\hline
\end{tabular}

b

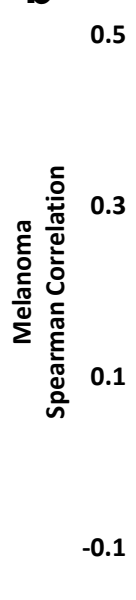

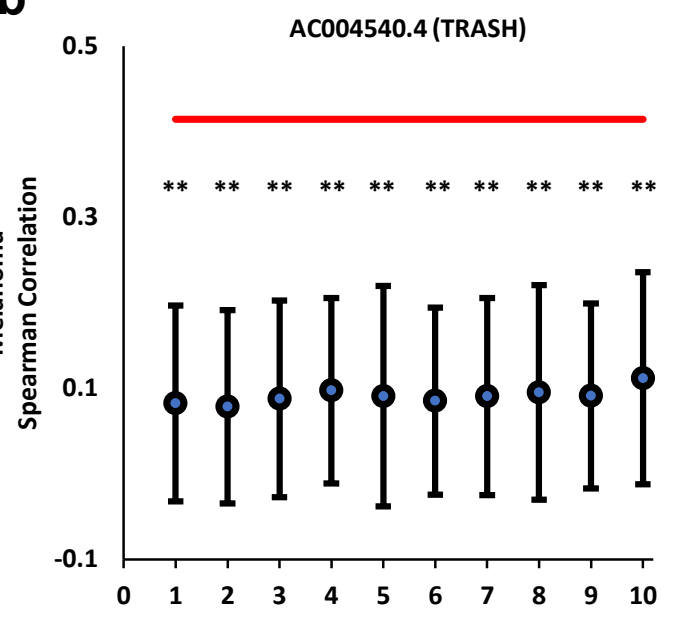

- TRASH correlations with 200 random genes

TRASH-hnR correlation

d

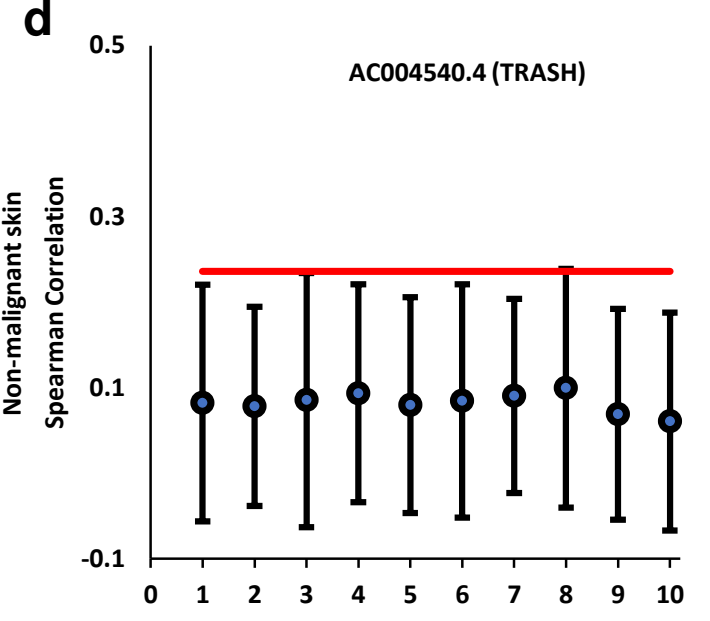

- TRASH correlations with 200 random genes

TRASH-hnR correlation

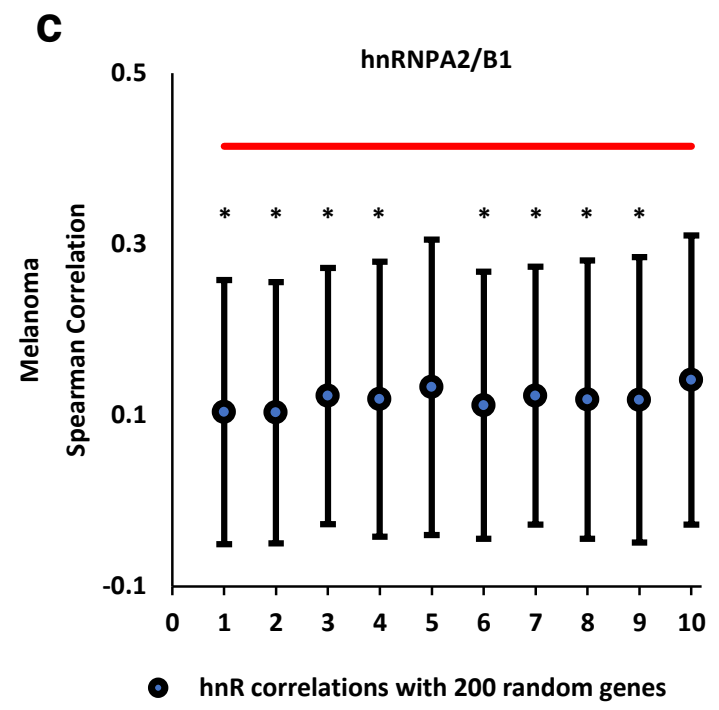

TRASH-hnR correlation

\section{e}

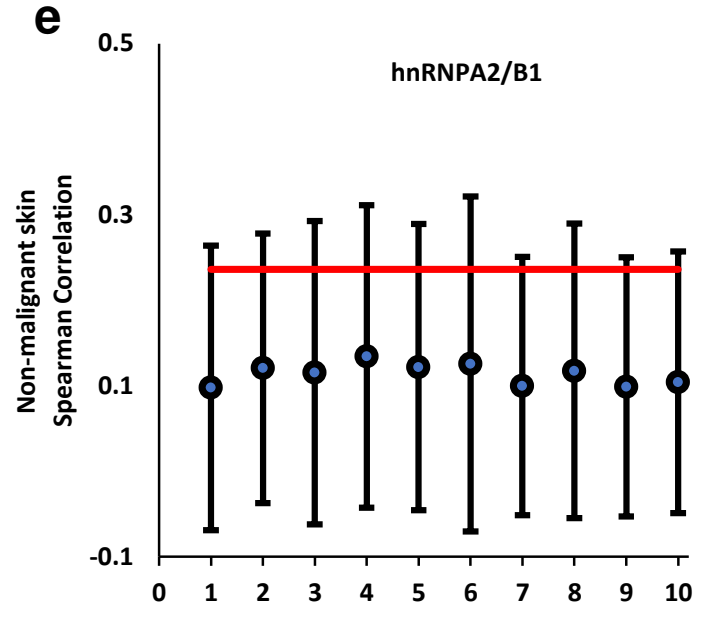

- TRASH correlations with 200 random genes 


\section{Supplementary Files}

This is a list of supplementary files associated with this preprint. Click to download.

- TRASHSupplementaryTable1.xlsx

- TRASHSupplementaryTable2.pdf

- TRASHSupplementaryTable3.xlsx 